FT/UCM-60-2000

\title{
The BRS invariance of noncommutative $U(N)$ Yang-Mills theory at the one-loop level
}

\author{
C. P. Martín* and D. Sánchez-Ruiz† \\ Departamento de Física Teórica I, Universidad Complutense, 28040 Madrid, Spain
}

\begin{abstract}
We show that $U(N)$ Yang-Mills theory on noncommutative Minkowski space-time can be renormalized, in a BRS invariant way, at the one-loop level, by multiplicative dimensional renormalization of its coupling constant, its gauge parameter and its fields. It is shown that the Slavnov-Taylor equation, the gauge-fixing equation and the ghost equation hold, up to order $\hbar$, for the MS renormalized noncommutative $U(N)$ Yang-Mills theory. We give the value of the pole part of every 1PI diagram which is UV divergent.
\end{abstract}

\section{1.- Introduction}

Noncommutative field theories occur both in the ordinary (commutative space-time) field theory setting and in the realm of string theory. The study of the large $\mathrm{N}$ limit of ordinary field theories naturally leads to field theories over noncommutative space $[1,2]$. General relativity and Heisenberg's uncertainty principles give rise, when strong gravitational fields are on, to space-times defined by noncommuting operators [3], whereupon it arises the need to define quantum field theories over noncommutative space-times. Super Yang-Mills theories on noncommutative tori occur in compactifications of M(atrix)theory [4], M(atrix)-theory on noncommutative tori being the subject of a good many papers [5]. Theories of strings ending on D-branes in the presence of a NS-NS B-field lead to noncommutative space-times; their infinite tension limit being -if unitarity allows itcertain noncommutative field theories [6]. It is therefore no wonder that a sizeable amount of work has been put in understanding, either in the continuum $[7,8]$ or on the lattice [9], whether quantum field theories make sense on noncommutative space-times. Applications to collider physics and Cosmology have just begun to come up [10].

Quantum field theories on noncommutative space-time present a characteristic connection between UV and IR scales: the virtual high-momenta modes contributing to a given Green function yield, when moving around a planar loop, an UV divergence, but

\footnotetext{
* email: carmelo@elbereth.fis.ucm.es

$\dagger$ email: domingos@eucmos.sim.ucm.es
} 
give rise to an IR divergence -even if the classical Lagrangian has only massive fields- as they propagate along a nonplanar loop. This is the UV/IR mixing unveiled in ref. [11], which has been further investigated in refs. [12]. The new -as regards to quantum field theory on commutative space-time- IR divergences that occur in noncommutative field theories makes it impossible [13], beyond a few loops, that these theories be renormalizable á la Bologiubov-Parasiuk-Hepp-Zimmerman [14], if supersymmetry is not called in [15]. Besides, they lack a Wilsonian action [11], which puts in jeopardy the implementation in noncommutative field theories of Wilson's renormalization group program [16]; and, hence, the existence of a continuum limit for these theories. The existence of a continuum limit for noncommutative field theories has been studied in ref. [17].

It is well known [7] that, at the one-loop level, only if a diagram is planar it can be UV divergent and that the momentum structure of this divergence, should it exist, is the product of a polynomial of the appropriate dimension of the external momenta (the UV degree of divergence of the Feynman loop integral) by suitable Moyal phases. Besides, if the noncommutative diagram has an ordinary counterpart (the diagram when space-time is commutative), the polynomial of the external momenta which carries the UV divergence is the same for both diagrams. This might lead us to think that noncommutative field theories are always one-loop renormalizable, if their ordinary counterpart is; which would in turn render almost trivial the issue of the one-loop renormalizability of noncommutative field theories. One cannot be more mistaken. There are certain $*$-deformations of $\lambda \phi^{4}$ that are shown not to be renormalizable at the one-loop level: see ref. [18].

It is common lore that $U(N)$ Yang-Mills theories on noncommutative Minowski are one-loop renormalizable. Indeed, if one assumes that gauge, better, BRS invariance is preserved at the one-loop level, it is difficult to think otherwise. However, statements about the BRS invariance of a field theory are rigorous only if they are based either on explicit computations or on the Quantum Action Principle [19] plus BRS cohomology techniques [20]. Since we lack a Quantum Action Principle for noncommutative field theories, we should better carry out explicit computations, lest our statements will be erected on shaky ground. Even if we had a Quantum Action Principle at our disposal, it would always be advisable to check general results by performing explicit computations up to a few loops.

In this paper we shall compute the complete UV divergent contribution to the 1PI functional of 4-dimensional noncommutative $U(N)$ Yang-Mills field theory for an arbitrary Lorentz gauge-fixing condition. We shall use dimensional regularization to carry out the computations. We shall thus show by explicit computation that this 1PI functional is the sum of two integrated polynomials (with respect to the Moyal product) of the field and its derivatives. The first term is the noncommutative Yang-Mills action. This term is nontrivial in the cohomology of the noncommutative Slavnov-Taylor operator and gives rise to the renormalization of the coupling constant. The second term is exact in the cohomology of the noncommutative Slavnov-Taylor operator and gives rise to the wave function and gauge-fixing parameter renormalizations. This result constitutes a highly nontrivial check of the one-loop BRS invariance of the the theory; the high nontriviality of the check stemming from the fact that the UV divergence of each planar diagram contributing to the 4-point function of the gauge field has a structure which differs very much from that of the 4-point tree-level contribution. The BRS invariance of the MS 
(minimal subtraction) UV divergent part of the one-loop 1PI functional leads, as we shall see, to a renormalized BRS invariant 1PI functional up to order $\hbar$.

The layout of this paper is as follows. In Section 2, we set the notation and display the Feynman rules for noncommutative $U(N)$ Yang-Mills field theory in an arbitrary Lorentz gauge. In Section 3, we give the UV divergent divergent contribution to the one-loop 1PI Green functions in the MS scheme of dimensional regularization and, from these data we obtain the MS UV divergent part of 1PI functional written in an explicitly BRS invariant form. Section 4 is devoted to comments and conclusions. In the Appendices we give for the record the UV divergent contribution to each one-loop 1PI diagram in the MS scheme.

\section{2.- Notation and Feynman rules}

The classical $U(N)$ field theory on noncommutative Minkowski space-time is given by the Yang-Mills functional

$$
S_{Y M}=-\frac{1}{4 g^{2} T_{R}} \int \operatorname{Tr}\left(F_{\mu \nu} \star F^{\mu \nu}\right)(x),
$$

where $F_{\mu \nu}(x)$ is given by

$$
F_{\mu \nu}(x)=\partial_{\mu} A_{\mu}-\partial_{\nu} A_{\mu}-i\left\{A_{\mu}, A_{\nu}\right\}(x)
$$

with $\left\{A_{\mu}, A_{\nu}\right\}(x)=\left(A_{\mu} \star A_{\nu}\right)(x)-\left(A_{\nu} \star A_{\mu}\right)(x)$.

The gauge field, $A_{\mu}$, is an $N \times N$ hermitian matrix, $\left(A_{\mu}\right)_{j}^{i}=\sum_{a=0}^{N^{2}-1} A_{\mu}^{a}\left(T_{a}\right)_{j}^{i}$. We shall take the hermitian $U(N)$ generators, $T^{a}$, normalized so that $\operatorname{Tr} T_{a} T_{b}=T_{R} \delta_{a b}$, if $a, b \geq 1, T_{0}=t_{\mathrm{R}} 1_{N \times N}$ with $\left(t_{\mathrm{R}}\right)^{2} N=T_{R}$. $A_{\mu}^{a}$ is a real vector function on $\mathbb{R}^{4}$. The symbol $*$ denotes the Moyal, or star, product defined as follows

$$
(f \star g)(x)=\iint \frac{d^{4} q}{(2 \pi)^{4}} \frac{d^{4} p}{(2 \pi)^{4}} e^{i(q+p)} e^{i \frac{1}{2} \theta^{\mu \nu} q_{\mu} p_{\nu}} f(q) g(p) .
$$

Here $f(q)$ and $q(p)$ are, respectively, the Fourier transforms of $f(x)$ and $g(x)$, the latter being two rapidly decreasing functions at infinity. $\theta^{\mu \nu}$ will be taken either magnetic or light-like, thus unitarity holds [21]. The reader is referred to ref. [22] for introductions to Noncommutative geometry. We shall denote $\frac{1}{2} \theta^{\mu \nu} p_{\mu} q_{\nu}$ by $\omega(p, q)$.

To quantize the classical noncommutative $U(N)$ Yang-Mills theory we shall use the path integral defined by means of the BRS quantization method. We introduce then the noncommutative ghost field, $c$, the noncommutative antighost field, $\bar{c}$, and the auxiliary field $B$. The fields $c, \bar{c}$ and $B$ are functions on $\mathbb{R}^{4}$ with values on the Lie algebra of $U(N)$. The BRS transformations read thus

$$
s A_{\mu}(x)=D_{\mu} c(x)=\partial_{\mu} c(x)-i\left\{A_{\mu}, c\right\}(x), s \bar{c}(x)=b, s b(x)=0, s c(x)=i(c \star c)(x) .
$$

The renormalization of the composite transformations $s A_{\mu}(x)$ and $s c(x)$ demands that the external fields $J_{\mu}(x)$ and $H(x)$, which couple to them, be ushered in. The BRS invariant 4-dimensional classical action for an arbitrary Lorentz gauge-fixing condition reads

$$
S_{\mathrm{cl}}=S_{\mathrm{YM}}+S_{\mathrm{gf}}+S_{\mathrm{ext}},
$$


where $S_{Y M}$ has been given in eq. (1) and

$$
\begin{aligned}
S_{\mathrm{gf}} & =\frac{1}{T_{R}} \int d^{4} x \operatorname{Tr} s\left[\bar{c} \star\left(\frac{\lambda}{2} B+\partial_{\mu} A^{\mu}\right)\right](x), \\
S_{\mathrm{ext}} & =\int d^{4} x \operatorname{Tr}\left(J^{\mu} \star s A_{\mu}+H \star s c\right)(x),
\end{aligned}
$$

where $\lambda$ is the gauge-fixing parameter.

Taking into account that

$$
\sum_{a=0}^{N^{2}-1}\left(T^{a}\right)_{i_{1}}^{j_{1}}\left(T^{a}\right)_{i_{2}}^{j_{2}}=T_{R} \delta_{i_{2}}^{j_{1}} \delta_{i_{1}}^{j_{2}}
$$

one readily shows that the gauge field propagator reads

$$
\int \frac{d^{4} p}{(2 \pi)^{4}} e^{-i p\left(x_{1}-x_{2}\right)} \frac{(-i)}{p^{2}} T_{R} g^{2} \delta_{i_{1}}^{j_{2}} \delta_{i_{2}}^{j_{1}}\left[g^{\mu_{1} \mu_{2}}+\left(\lambda^{\prime}-1\right) \frac{p^{\mu_{1}} p^{\mu_{2}}}{p^{2}}\right]
$$

where $\lambda^{\prime} \equiv \lambda / g^{2}$. The remaining propagators and tree-level vertices are obtained from eq. (2) by using standard path integral techniques. We have gathered the Feynman rules in Figures 1 and 2. Note that the Feynman rules are rendered in 't Hooft's double index notation [23]. This notation is very appropriate to work out the colour contribution to a given $U(N)$ Yang-Mills diagram.

For the Noncommutative $U(N)$ gauge theory to be BRS invariant at the quantum level the Slavnov-Taylor identity for the 1PI functional $\Gamma\left[A_{\mu}, B, c, \bar{c} ; J_{\mu}, H\right]$ must hold. This identity reads

$$
\mathcal{S}(\Gamma) \equiv \int d^{4} x \operatorname{Tr}\left[\frac{\delta \Gamma}{\delta J^{\mu}} \frac{\delta \Gamma}{\delta A_{\mu}}+\frac{\delta \Gamma}{\delta H} \frac{\delta \Gamma}{\delta c}+B \frac{\delta \Gamma}{\delta \bar{c}}\right]=0
$$

For the one-loop, $\Gamma_{1}$, contribution to $\Gamma\left[A_{\mu}, B, c, \bar{c} ; J_{\mu}, H\right]$, the Slavnov-Taylor equation boils down to

$$
\mathcal{B} \Gamma_{1}=0
$$

where

$$
\mathcal{B}=\int d^{4} x \operatorname{Tr}\left[\frac{\delta S_{\mathrm{cl}}}{\delta J^{\mu}} \frac{\delta}{\delta A_{\mu}}+\frac{\delta S_{\mathrm{cl}}}{\delta A^{\mu}} \frac{\delta}{\delta J_{\mu}}+\frac{\delta S_{\mathrm{cl}}}{\delta H} \frac{\delta}{\delta c}+\frac{\delta S_{\mathrm{cl}}}{\delta c} \frac{\delta}{\delta H}+B \frac{\delta}{\delta \bar{c}}\right] .
$$

$\mathcal{B}$ is the linearized noncommutative Slavnov-Taylor operator.

Since the formal Feynman diagrams contributing to $\Gamma\left[A_{\mu}, B, c, \bar{c} ; J_{\mu}, H\right]$ present UV divergences, it is not straightforward that the renormalized, would it exist, 1PI functional defining the quantum theory satisfies the Slavnov-Taylor identity: anomalies may occur. We shall see in this paper that at the one-loop level the theory at hand can be renormalized in such a way that eq. (4) holds for the MS renormalized action. 


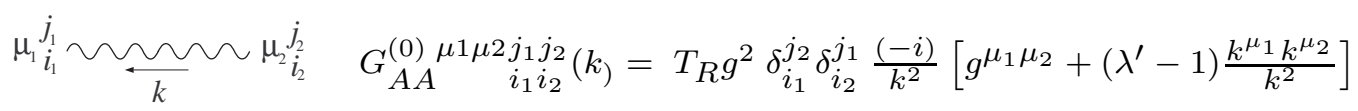

$\sum_{k_{1}}^{\mu_{1}^{j_{1}}} \sum_{i_{2}}^{j_{i_{2}}}$

$i S_{A A A}^{\mu_{1} \mu_{2} \mu_{3} j_{1} j_{2} j_{3}}{ }_{i_{1} i_{2} i_{3}}\left(k_{1}, k_{2}, k_{3}=-k_{1}-k_{2}\right)=$

$\frac{i}{g^{2} T_{R}}\left[\delta_{i_{1}}^{j_{3}} \delta_{i_{2}}^{j_{1}} \delta_{i_{3}}^{j_{2}} e^{i \omega\left(k_{1}, k_{2}\right)}-\delta_{i_{1}}^{j_{2}} \delta_{i_{2}}^{j_{3}} \delta_{i_{3}}^{j_{1}} e^{-i \omega\left(k_{1}, k_{2}\right)}\right]$

$\left[g^{\mu_{1} \mu_{2}}\left(k_{1}-k_{2}\right)^{\mu_{3}}+g^{\mu_{2} \mu_{3}}\left(k_{2}-k_{3}\right)^{\mu_{1}}+g^{\mu_{3} \mu_{1}}\left(k_{3}-k_{1}\right)^{\mu_{2}}\right]$

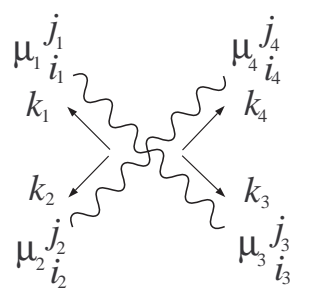

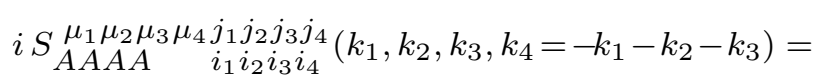

$\frac{i}{g^{2} T_{R}} \delta_{i_{1}}^{j_{4}} \delta_{i_{2}}^{j_{1}} \delta_{i_{3}}^{j_{2}} \delta_{i_{4}}^{j_{3}} e^{i\left[\omega\left(k_{1}, k_{2}\right)+\omega(k 3, k 4)\right]}$

$\left(2 g^{\mu_{1} \mu_{3}} g^{\mu_{2} \mu_{4}}-g^{\mu_{1} \mu_{4}} g^{\mu_{2} \mu_{3}}-g^{\mu_{1} \mu_{2}} g^{\mu_{3} \mu_{4}}\right)$

$+(1243)+(1324)+(1342)+(1423)+(1432)$
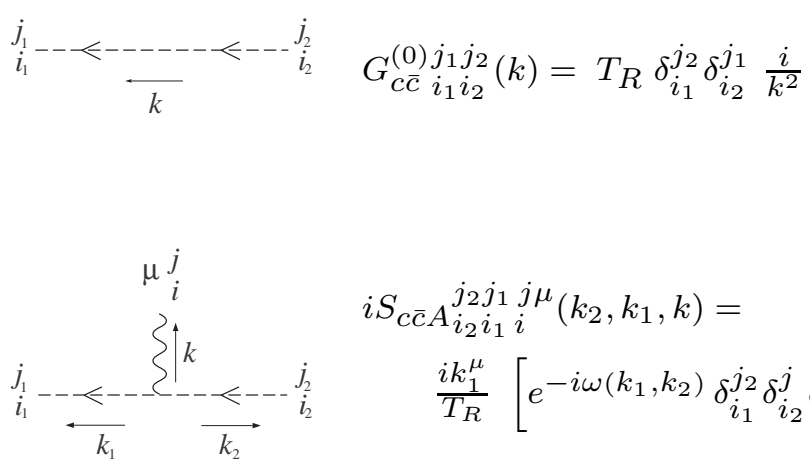

$$
\begin{aligned}
& i S_{c \bar{c} A_{i_{2}} i_{1}}^{j_{2} j_{1} j \mu}\left(k_{2}, k_{1}, k\right)= \\
& \frac{i k_{1}^{\mu}}{T_{R}}\left[e^{-i \omega\left(k_{1}, k_{2}\right)} \delta_{i_{1}}^{j_{2}} \delta_{i_{2}}^{j} \delta_{i}^{j_{1}}-e^{i \omega\left(k_{1}, k_{2}\right)} \delta_{i_{1}}^{j} \delta_{i_{2}}^{j_{1}} \delta_{i}^{j_{2}}\right]
\end{aligned}
$$

Figure 1: Feynman rules for noncommutative $U(N)$ Yang-Mills theory: Propagators and vertices with no external fields. $(i j k l)$ denotes a permutation of (1 234 ).

To regularize the Feynman integrals of our theory will shall use dimensional regularization. To define $\theta^{\mu \nu}$ in dimensional regularization we shall follow the philosophy in ref. [24] and say that $\theta^{\mu \nu}$ is an algebraic object which satisfies the following equations

$$
\theta^{\mu \nu}=-\theta^{\nu \mu}, \hat{\eta}_{\mu \rho} \theta^{\rho \nu}=0, p_{\mu} \theta^{\mu \rho} \eta_{\rho \sigma} \theta^{\sigma \nu} p_{\nu} \geq 0, \forall p_{\mu}
$$

Here $\eta_{\rho \sigma}$ and $\hat{\eta}_{\mu \rho}$ are, respectively, the "D-dimensional" and "D-4-dimensional" metrics as defined in ref. [24]. It is not difficult to convince oneself that, with the previous definition of $\theta_{\mu \nu}$, the one-loop Feynman integrals do have a mathematically well-defined expressions and that the techniques used in ref. [24] to prove the Quantum Action Principle for dimensionally regularized ordinary field theories can also be employed here to conclude that at 


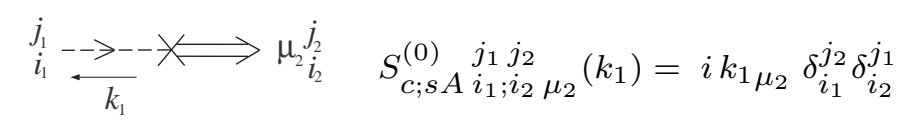

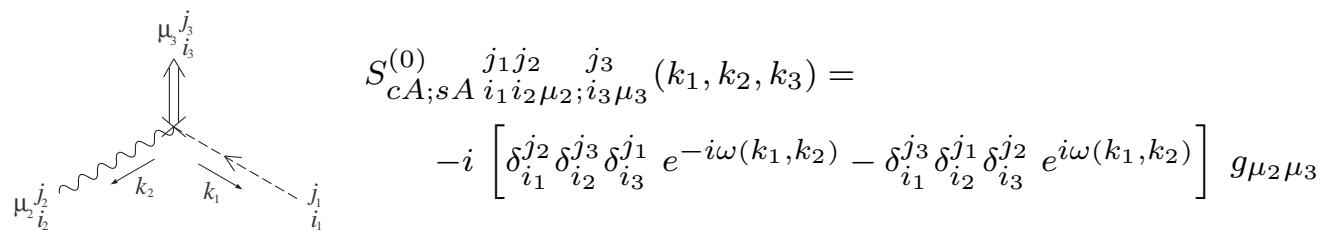

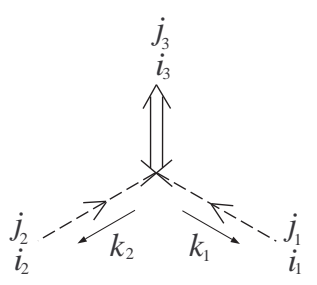

$$
\begin{aligned}
& S_{c c ; s c}^{(0)}{ }^{j_{1} j_{2} j_{3}} i_{i_{1} i_{2} ; i_{3}}\left(k_{1}, k_{2}, k_{3}\right)= \\
& \quad i\left[\delta_{i_{1}}^{j_{2}} \delta_{i_{2}}^{j_{3}} \delta_{i_{3}}^{j_{1}} e^{-i \omega\left(k_{1}, k_{2}\right)}-\delta_{i_{1}}^{j_{3}} \delta_{i_{2}}^{j_{1}} \delta_{i_{3}}^{j_{2}} e^{i \omega\left(k_{1}, k_{2}\right)}\right]
\end{aligned}
$$

Figure 2: Feynman rules for noncommutative $U(N)$ Yang-Mills theory: Vertices with insertions of BRS variations.

the one-loop level our dimensionally regularized noncommutative $U(N)$ is BRS invariant. The so regularized Feynman diagrams are meromorphic functions of $D$, with simple poles at $D=4$, if they are planar, and no poles, if they are nonplanar and $P_{\mu} \theta^{\mu \rho} \eta_{\rho \sigma} \theta^{\sigma \nu} P_{\nu}>0$ for any linear combination, $P$, of the external momenta.

\section{3.- The MS UV divergent part of the 1PI functional}

The fact that at the one-loop level only planar diagrams can be UV divergent and the fact that planar diagrams have the same UV degree as their ordinary field theory counterparts readily leads to the conclusion that the one-loop UV divergent 1PI functions are following: $\Gamma_{A A}, \Gamma_{A A A}, \Gamma_{A A A A}, \Gamma_{\bar{c} c}, \Gamma_{\bar{c} A c}, \Gamma_{J c}, \Gamma_{J A c}$ and $\Gamma_{H c c}$, with obvious notation.

We have computed the one-loop UV divergent contribution to all the divergent 1PI Green functions. Taking into account the results presented in the Appendices, one obtains the following values for these UV divergent part in the MS scheme:

$$
\begin{aligned}
& \Gamma_{\mu_{1} \mu_{2}}^{(A A),(\text { pole })} \underset{i_{1} i_{2}}{j_{1} j_{2}}(p)=\left(\frac{1}{(4 \pi)^{2} \varepsilon}\right)\left(\frac{10}{3}-\left(\lambda^{\prime}-1\right)\right) N \delta_{i_{1}}^{j_{2}} \delta_{i_{2}}^{j_{1}}\left(p^{2} g_{\mu_{1} \mu_{2}}-p_{\mu_{1}} p_{\mu_{2}}\right) \\
& \Gamma_{\mu_{1} \mu_{2} \mu_{3}}^{(A A A),(\text { pole }) j_{1} j_{1} j_{2} j_{3} i_{3}}\left(p_{1}, p_{2}, p_{3}\right)=\left(\frac{1}{(4 \pi)^{2} \varepsilon}\right)\left(\frac{4}{3}-\frac{3}{2}\left(\lambda^{\prime}-1\right)\right) \\
& N\left[e^{-i \omega\left(p_{1}, p_{2}\right)} \delta_{i_{1}}^{j_{2}} \delta_{i_{2}}^{j_{3}} \delta_{i_{3}}^{j_{1}}-e^{i \omega\left(p_{1}, p_{2}\right)} \delta_{i_{1}}^{j_{3}} \delta_{i_{2}}^{j_{1}} \delta_{i_{3}}^{j_{2}}\right] \\
& \left(\left(p_{1}-p_{2}\right)_{\mu_{3}} g_{\mu_{1} \mu_{2}}+\left(p_{2}-p_{3}\right)_{\mu_{1}} g_{\mu_{2} \mu_{3}}+\left(p_{3}-p_{1}\right)_{\mu_{2}} g_{\mu_{1} \mu_{3}}\right)
\end{aligned}
$$




$$
\begin{aligned}
& \Gamma_{\mu_{1} \mu_{2} \mu_{3} \mu_{4}}^{(A A A A),(\text { pole }){ }_{i_{1} i_{2} i_{3} j_{4}} j_{2} j_{3} j_{4}}\left(p_{1}, p_{2}, p_{3}, p_{4}\right)=\left(\frac{1}{(4 \pi)^{2} \varepsilon}\right)\left(\frac{2}{3}+2\left(\lambda^{\prime}-1\right)\right) \\
& N \delta_{i_{1}}^{j_{4}} \delta_{i_{2}}^{j_{1}} \delta_{i_{3}}^{j_{2}} \delta_{i_{4}}^{j_{3}} e^{i\left[\omega\left(p_{1}, p_{2}\right)+\omega\left(p_{3}, p_{4}\right)\right]} \\
& \left(2 g_{\mu_{1} \mu_{3}} g_{\mu_{2} \mu_{4}}-g_{\mu_{1} \mu_{4}} g_{\mu_{2} \mu_{3}}-g_{\mu_{1} \mu_{2}} g_{\mu_{3} \mu_{4}}\right)+ \\
& (1243)+(1324)+(1342)+(1423)+(1432)
\end{aligned}
$$

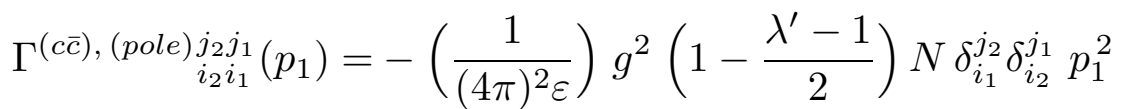

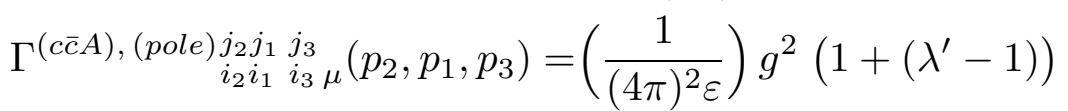

$$
\begin{aligned}
& N\left[e^{-i \omega\left(p_{1}, p_{2}\right)} \delta_{i_{1}}^{j_{2}} \delta_{i_{2}}^{j_{3}} \delta_{i_{3}}^{j_{1}}-e^{i \omega\left(p_{1}, p_{2}\right)} \delta_{i_{1}}^{j_{3}} \delta_{i_{2}}^{j_{1}} \delta_{i_{3}}^{j_{2}}\right] p_{1 \mu}
\end{aligned}
$$

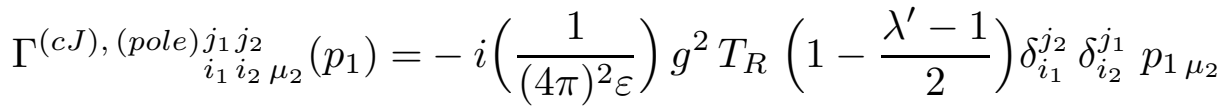

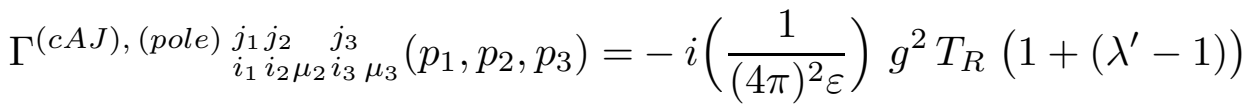

$$
\begin{aligned}
& N\left[e^{-i \omega\left(p_{1}, p_{2}\right)} \delta_{i_{1}}^{j_{2}} \delta_{i_{2}}^{j_{3}} \delta_{i_{3}}^{j_{1}}-e^{i \omega\left(p_{1}, p_{2}\right)} \delta_{i_{1}}^{j_{3}} \delta_{i_{2}}^{j_{1}} \delta_{i_{3}}^{j_{2}}\right] g_{\mu_{2} \mu_{3}} \\
& \Gamma^{(c c H),(\text { pole }) j_{1} j_{1} j_{2} j_{3}}\left(p_{1}, p_{2}, p_{3}\right)=i\left(\frac{1}{(4 \pi)^{2} \varepsilon}\right) g^{2} T_{R}\left(1+\left(\lambda^{\prime}-1\right)\right) \\
& N\left[e^{-i \omega\left(p_{1}, p_{2}\right)} \delta_{i_{1}}^{j_{2}} \delta_{i_{2}}^{j_{3}} \delta_{i_{3}}^{j_{1}}-e^{i \omega\left(p_{1}, p_{2}\right)} \delta_{i_{1}}^{j_{3}} \delta_{i_{2}}^{j_{1}} \delta_{i_{3}}^{j_{2}}\right],
\end{aligned}
$$

where $D=4-2 \varepsilon,(i j k l)$ stands for the corresponding permutation of the indices and the following convention has been taken, in order to take properly account of signs:

$$
\Gamma_{i_{1} i_{2} \ldots i_{n}}^{\left(\Phi_{1} \Phi_{2} \ldots \Phi_{n}\right)}=\left.\frac{\delta \Gamma}{\delta \Phi_{1}^{i_{1}} \delta \Phi_{2}^{i_{2}} \ldots \delta \Phi_{n}^{i_{n}}}\right|_{0}, \quad \Gamma_{i_{1} i_{2} \ldots i_{n} i}^{\left(\phi_{1} \phi_{2} \ldots \phi_{n} K_{\phi}\right)}=\left.\frac{\delta s \phi_{i} \cdot \Gamma}{\delta \phi_{1}^{i_{1}} \delta \phi_{2}^{i_{2}} \ldots \delta \phi_{n}^{i_{n}}}\right|_{0},
$$

with $\Phi$ standing for any, internal or external, field and $K_{\phi}$ for the external field coupled to $s \phi$. The already known results for the $U(1)$ theory are retrieved by setting $N=T_{R}=1$ in the previous expressions.

Notice that, upon formal generalization of $S_{\mathrm{cl}}$ in eq. (2) to the " $D$-dimensional" space of dimensional regularization, the momentum structure of the singular contributions displayed above is the same as the corresponding term in $S_{\mathrm{cl}}$. So, one would expect that these 1PI contributions can be subtracted by MS (minimal subtraction) multiplicative renormalization of the fields and parameters in the BRS invariant action. And, indeed, this is so, if we perform the following infinite renormalizations

$$
\begin{aligned}
& g_{0}=\mu^{2 \varepsilon} Z_{g} g, \lambda_{0}=Z_{\lambda} \lambda, A_{0 \mu}=Z_{A} A_{\mu}, B_{0}=Z_{B} B, \\
& J_{0 \mu}=Z_{J} J_{\mu}, H_{0}=Z_{H} H, c_{0}=Z_{c} c \text { and } \bar{c}_{0}=Z_{\bar{c}} \bar{c},
\end{aligned}
$$

where the subscript 0 labels the bare quantities. Now,

$$
\begin{array}{r}
S_{Y M}^{0}=S_{Y M}-Z_{g}^{(1)}\left(2 S_{A A}+2 S_{A A A}+2 S_{A A A A}\right)+ \\
Z_{A}^{(1)}\left(2 S_{A A}+3 S_{A A A}+4 S_{A A A A}\right),
\end{array}
$$


so that

$$
\begin{aligned}
& -2 Z_{g}^{(1)}+2 Z_{A}^{(1)}=\frac{1}{(4 \pi)^{2} \varepsilon} 2 N\left[\frac{5}{3}-\frac{1}{2}\left(\lambda^{\prime}-1\right)\right] g^{2} T_{R}, \\
& -2 Z_{g}^{(1)}+3 Z_{A}^{(1)}=\frac{1}{(4 \pi)^{2} \varepsilon} 2 N\left[\frac{2}{3}-\frac{3}{4}\left(\lambda^{\prime}-1\right)\right] g^{2} T_{R}, \\
& -2 Z_{g}^{(1)}+4 Z_{A}^{(1)}=\frac{1}{(4 \pi)^{2} \varepsilon} 2 N\left[-\frac{1}{3}-\left(\lambda^{\prime}-1\right)\right] g^{2} T_{R} .
\end{aligned}
$$

Hence,

$$
Z_{g}=1-\frac{1}{(4 \pi)^{2} \varepsilon} 2 N \frac{11}{6} g^{2} T_{R}, Z_{A}=1-\frac{1}{(4 \pi)^{2} \varepsilon} 2 N\left[1+\frac{1}{4}\left(\lambda^{\prime}-1\right)\right] g^{2} T_{R}
$$

Analogously,

$$
\begin{aligned}
& Z_{\bar{c}} Z_{c}=1+\frac{1}{(4 \pi)^{2} \varepsilon} \frac{3-\lambda^{\prime}}{2} g^{2} T_{R} N, Z_{\bar{c}} Z_{A} Z_{c}=1-\frac{1}{(4 \pi)^{2} \varepsilon} \lambda^{\prime} g^{2} T_{R} N \\
& Z_{H} Z_{c}^{2}=1-\frac{1}{(4 \pi)^{2} \varepsilon} \lambda^{\prime} g^{2} T_{R} N, Z_{B}=Z_{A}^{-1}, Z_{\lambda}=Z_{A}^{2} \quad \text { and } \quad Z_{J}=Z_{\bar{c}} .
\end{aligned}
$$

That the $Z \mathrm{~s}$ in eqs. (7) and (8) render UV finite the one-loop 1PI functional is a consequence of BRS invariance. Indeed, in view of eq. (6), it is not difficult to show that the singular contribution, $\Gamma^{(\text {pole })}$, to the dimensionally regularized 1PI functional can be cast into the form

$$
\Gamma^{(\text {pole })}=\frac{a}{4 g^{2} T_{R}} \int d^{D} x \operatorname{Tr}\left(F_{\mu \nu} \star F^{\mu \nu}\right)(x)+\mathcal{B}_{D} X,
$$

where

$$
\begin{gathered}
X=\int d^{D} x \operatorname{Tr}\left(a_{1}\left(J_{\mu}+\partial_{\mu} \bar{c}\right) \star A_{\mu}-a_{2} H \star c\right)(x), \\
a=\frac{1}{(4 \pi)^{2} \varepsilon} \frac{22}{3} N T_{R} g^{2}, a_{1}=+\frac{1}{(4 \pi)^{2} \varepsilon} \frac{3+\lambda^{\prime}}{2} N T_{R} g^{2}, a_{2}=+\frac{1}{(4 \pi)^{2} \varepsilon} \lambda^{\prime} N T_{R} g^{2},
\end{gathered}
$$

and $\mathcal{B}_{D}$ is the linearized Slavnov-Taylor operator acting upon the space of formal *polynomials constructed with " $D$-dimensional" monomials of the fields and their derivatives. $\mathcal{B}_{D}$ is defined as follows

$$
\mathcal{B}_{D}=\int d^{D} x \operatorname{Tr}\left[\frac{\delta S_{\mathrm{cl}}}{\delta J^{\mu}} \frac{\delta}{\delta A_{\mu}}+\frac{\delta S_{\mathrm{cl}}}{\delta A^{\mu}} \frac{\delta}{\delta J_{\mu}}+\frac{\delta S_{\mathrm{cl}}}{\delta H} \frac{\delta}{\delta c}+\frac{\delta S_{\mathrm{cl}}}{\delta c} \frac{\delta}{\delta H}+B \frac{\delta}{\delta \bar{c}}\right] .
$$

The operator $\mathcal{B}_{D}$ is the " $D$-dimensional" counterpart of the operator $\mathcal{B}$ defined in eq. (5).

Eq. (9) gives, in an explicitly BRS invariant form, the UV divergent contribution to the 1PI functional in the MS scheme of Dimensional Regularization. Note that $\Gamma^{(\text {pole })}$ is 
the sum of two terms: the first term, the Yang-Mills term, is $\mathcal{B}_{D}$-closed and the second term is $\mathcal{B}_{D}$-exact (recall that $\mathcal{B}_{D}^{2}=0$ ). The analogy with ordinary $S U(N)$ Yang-Mills theory is apparent. And, indeed, as as in ordinary four-dimensional Yang-Mills theory we have

$$
\begin{aligned}
& Z_{g}=1-\frac{a}{2}, Z_{A}=1+a_{1}, Z_{\bar{c}} Z_{c}=1-a_{1}+a_{2}, Z_{\bar{c}} Z_{A} Z_{c}=1+a_{2}, \\
& Z_{H} Z_{c}^{2}=1+a_{2}, Z_{B}=Z_{A}^{-1}, Z_{\lambda}=Z_{A}^{2} \quad \text { and } \quad Z_{J}=Z_{\bar{c}}
\end{aligned}
$$

Eqs. (7) and (8) are thus retrieved. Let us remark that the values we have obtained for the $Z$ s agree with the corresponding values of the $Z$ s of $S U(N)$ Yang-Mills theory on ordinary Minkowski space-time.

We shall define as usual the renormalized one-loop $1 \mathrm{PI}$ functional, $\Gamma_{\text {ren }}^{(1), \mathrm{MS}}$, in the MS scheme:

$$
\Gamma_{\text {ren }}^{(1), M S}=\mathcal{L} \mathcal{I} \mathcal{M}_{\varepsilon \rightarrow 0}\left[\Gamma_{\text {DReg }}^{(1)}-\Gamma^{(\text {pole })}\right] .
$$

Here $\Gamma_{\text {DReg }}^{(1)}$ denotes the dimensionally regularized 1PI functional at order $\hbar$ and the functional $\Gamma^{(\text {pole })}$ is given in eq. (9). The limit $\varepsilon \rightarrow 0$ is taken after performing the subtraction of the pole and replacing every " $D$-dimensional" algebraic object with its 4 -dimensional counterpart [24]; this is why we have denoted it by $\mathcal{L} \mathcal{I} \mathcal{M}$. We shall not discuss in this paper how to make sense out of the noncommutative IR divergences [11] that occur in $\Gamma_{\text {ren }}^{(1), M S}$.

Since $\Gamma_{\text {DReg }}^{(1)}$ is BRS invariant, i.e., it satisfies the Slavnov-Taylor identity at order $\hbar$

$$
\mathcal{B}_{D} \Gamma_{\mathrm{DReg}}^{(1)}=0
$$

the MS renormalized 1PI functional $\Gamma_{\text {ren }}^{(1), M S}$ is also BRS invariant:

$$
\mathcal{B} \Gamma_{\text {ren }}^{(1), M S}=0
$$

The operator $\mathcal{B}$ has been defined in eq. (5). We thus conclude that the Slavnov-Taylor identity (eq. (3)) holds for the renormalized theory at order $\hbar$.

By using standard textbook techniques, one can work out the renormalization group equation -expressing the invariance of the observables under changes of the renormalization scale $\mu$ - for $\Gamma_{\text {ren }}^{\mathrm{MS}}$ :

$$
\left[\mu \frac{\partial}{\partial \mu}+\beta \frac{\partial}{\partial g^{2}}-\delta_{\lambda} \frac{\partial}{\partial \lambda}-\sum_{\phi} \gamma_{\phi} \int d^{4} x \phi(x) \frac{\delta}{\delta \phi(x)}\right] \Gamma_{\mathrm{ren}}^{\mathrm{MS}}[\phi ; g, \theta, \lambda]=0 .
$$

The fields are denoted by $\phi$. It should be noticed that $\theta_{\mu \nu}$ is a dimensionful parameter which is not renormalized in the MS renormalization scheme.

The one-loop beta function of the theory is easily computed to be

$$
\beta\left(g^{2}\right) \equiv \mu \frac{d g^{2}}{d \mu}=-\frac{1}{8 \pi^{2}} \frac{22}{3} N T_{R} g^{4}
$$


The other renormalization group coefficients read at the one-loop level:

$$
\begin{aligned}
& \gamma_{A}=+\frac{1}{8 \pi^{2}}\left(\frac{3+\lambda^{\prime}}{2}\right) N T_{R} g^{2}, \quad \gamma_{c}=+\frac{1}{8 \pi^{2}} \lambda^{\prime} N T_{R} g^{2}, \\
& \gamma_{J}=\gamma_{\bar{c}}=\gamma_{B}=-\gamma_{A}, \quad \delta_{\lambda}=-2 \gamma_{A} \lambda, \quad \gamma_{H}=-\gamma_{c} .
\end{aligned}
$$

In the proof of the renormalizability of ordinary $S U(N)$ Yang-Mills theory, besides the Slavnov-Taylor equation, two equations play an important role, namely, the gauge-fixing and ghost equation [20]. Do these equations also hold for noncommutative $U(N)$ YangMills theory? It is not difficult to show that $\Gamma^{(\text {pole })}$ in eq. (9) verifies both the gauge-fixing equation and the ghost equation:

$$
\frac{\delta \Gamma^{(\text {pole })}}{\delta B}=0, \quad \frac{\delta \Gamma^{(\text {pole })}}{\delta \bar{c}}+\partial_{\mu} \frac{\delta \Gamma^{(\text {pole })}}{\delta J_{\mu}}=0 .
$$

Hence, up to order $\hbar$, the MS renormalized 1PI functional does satisfy both the gauge-fixing equation and the ghost equation:

$$
\begin{gathered}
T_{R} \frac{\delta \Gamma_{\mathrm{ren}}^{\mathrm{MS}}}{\delta B}=\lambda B+\partial A+O\left(\hbar^{2}\right), \\
\frac{\delta \Gamma_{\mathrm{ren}}^{\mathrm{MS}}}{\delta \bar{c}}+\partial_{\mu} \frac{\delta \Gamma_{\mathrm{ren}}^{\mathrm{MS}}}{\delta J_{\mu}}=0+O\left(\hbar^{2}\right) .
\end{gathered}
$$

\section{4.- Conclusions and comments}

In this paper we have computed, within the MS scheme of dimensional regularization, all the one-loop UV divergent contributions to the 1PI functional for noncommutative $U(N)$ Yang-Mills theory in an arbitrary Lorentz gauge. We have shown that these contributions satisfy the Slavnov-Taylor equation, the gauge-fixing equation and the ghost equation and, hence, that the MS renormalized 1PI functional satisfies those equations as well. That the one-loop UV divergent contributions to the 1PI functional satisfy the Slavnov-Taylor equation has been shown by casting the pole part of the 1PI functional in an explicitly BRS invariant form. As with ordinary $S U(N)$ Yang-Mills theory, this explicitly BRS invariant form is the sum of a $\mathcal{B}$-closed term -which is proportional to the Yang-Mills action- and $\mathcal{B}$-exact term; being $\mathcal{B}$ the linearized noncommutative BRS operator. Our computations lead us to believe that the $*$-deformation of the ordinary BRS cohomology techniques [20] are to play an important role in the proof of the perturbative renormalizability of noncommutative gauge theories.

That the 2- and 3-point functions of noncommutative $U(N)$ theory can be renormalized in a BRS invariant way does not come as a surprise once one shows, as we have done in this paper, that the sum of a given diagram with its permutations, the diagrams being planar, is proportional to the tree-level 1PI Green function. This generalizes the results in refs. $[1,7]$. The reader is referred to the Appendices for further details; where he can realize, in particular, that every contribution listed there grows with $N$ as corresponds to the fact that they come only from planar diagrams. 
That the one-loop 4-point function of the gauge field has a MS UV divergent part which does not spoil the Slavnov-Taylor equation is, though, highly nontrivial. It demands that very delicate cancellations occur upon adding all the UV divergent 4-point diagrams: unlike 2- and 3-point diagrams, the sum of 4-point diagrams of the same type is not proportional to tree-level 4-point gauge vertex. That these delicate cancellations do happen is, of course, a consequence of the fact that BRS invariance holds indeed. In this regard, we invite the reader to go to Appendix $\mathrm{C}$ and eq. (6) and get acquainted with the momentum and colour structure of the UV divergent contributions reported there. Note that every 4-point UV divergent contribution has an overall factor equal to $N$, for they come only from planar diagrams.

As regards the actual value of the beta function, the anomalous dimensions of the fields and gauge-fixing parameter, we have found that they are those of $S U(N)$ 's. This result is, of course, almost trivial [7, 11], once it is shown that BRS holds. Indeed, all 1PI planar diagrams but the 4-point diagrams can be grouped in classes of planar diagrams of the same type, the sum of the diagrams in each class being proportional to the corresponding tree-level contributions. However, taking into account what it is at stake, computations which are both explicit and thorough are much welcomed.

Finally, the computations presented in this paper were finished more than a year ago. In the meantime two papers which overlap with it have appeared [25]. Our findings are in agreement with theirs, but ours are more general.

\section{Appendix A. Gauge field 2-point function}

The diagrams which are UV divergent in dimensional regularization are the planar diagrams in Figure 3. Note that the planar tadpole diagram is not singular at $D=4$ in dimensional regularization.

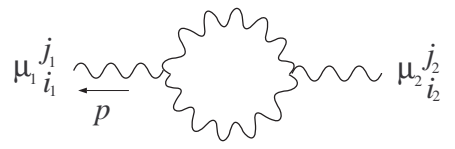

(i)

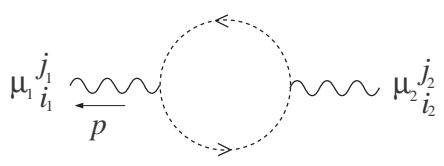

(ii)

Figure 3: 1PI UV divergent 2-point Feynman diagrams for the gauge field.

The minimal UV divergent part of each diagram reads

$$
\begin{aligned}
& (i)=2 i\left(\frac{1}{(4 \pi)^{2} \varepsilon}\right) N \delta_{i_{1}}^{j_{2}} \delta_{i_{2}}^{j_{1}}\left[\left(\frac{19}{12}-\frac{\left(\lambda^{\prime}-1\right)}{2}\right) p^{2} g_{\mu_{1} \mu_{2}}-\left(\frac{11}{6}-\frac{\left(\lambda^{\prime}-1\right)}{2}\right) p_{\mu_{1}} p_{\mu_{2}}\right] \\
& (i i)=2 i\left(\frac{1}{(4 \pi)^{2} \varepsilon}\right) N \delta_{i_{1}}^{j_{2}} \delta_{i_{2}}^{j_{1}}\left[\frac{1}{12} p^{2} g_{\mu_{1} \mu_{2}}+\frac{1}{6} p_{\mu_{1}} p_{\mu_{2}}\right] .
\end{aligned}
$$




\section{Appendix B. Gauge field 3-point function}

The UV divergent part of the 3-point function of the gauge field is obtained from the planar diagrams in Figure 4.

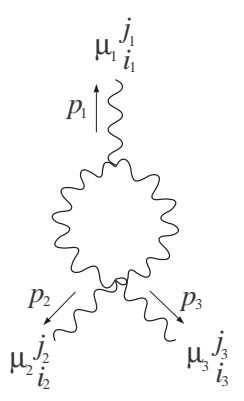

$(i) 123$

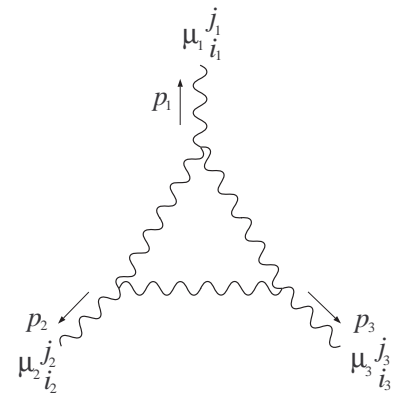

$($ ii $) 123$

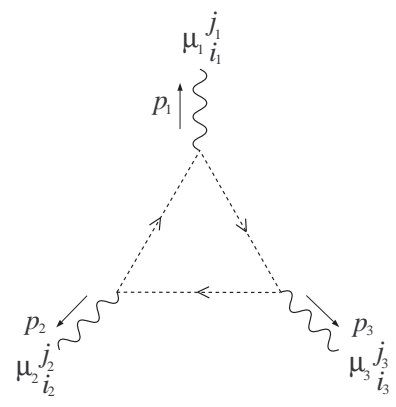

$(\text { iii })_{123}$

Figure 4: 1PI UV divergent 3-point Feynman diagrams for the gauge field.

The UV divergent part of these diagrams read

$$
\begin{aligned}
& (i)_{123}=i\left(\frac{1}{(4 \pi)^{2} \varepsilon}\right)\left(\frac{9}{2}+\frac{3\left(\lambda^{\prime}-1\right)}{4}\right) N\left[e^{-i \omega\left(p_{1}, p_{2}\right)} \delta_{i_{1}}^{j_{2}} \delta_{i_{2}}^{j_{3}} \delta_{i_{3}}^{j_{1}}-e^{i \omega\left(p_{1}, p_{2}\right)} \delta_{i_{1}}^{j_{3}} \delta_{i_{2}}^{j_{1}} \delta_{i_{3}}^{j_{2}}\right] \times \\
& \left(p_{1 \mu_{3}} g_{\mu_{1} \mu_{2}}-p_{1 \mu_{2}} g_{\mu_{1} \mu_{3}}\right) \text {, } \\
& (i i)_{123}=i\left(\frac{1}{(4 \pi)^{2} \varepsilon}\right)\left(\frac{13}{4}+\frac{9\left(\lambda^{\prime}-1\right)}{4}\right) N\left[e^{-i \omega\left(p_{1}, p_{2}\right)} \delta_{i_{1}}^{j_{2}} \delta_{i_{2}}^{j_{3}} \delta_{i_{3}}^{j_{1}}-e^{i \omega\left(p_{1}, p_{2}\right)} \delta_{i_{1}}^{j_{3}} \delta_{i_{2}}^{j_{1}} \delta_{i_{3}}^{j_{2}}\right] \times \\
& \left(\left(p_{2}-p_{1}\right)_{\mu_{3}} g_{\mu_{1} \mu_{2}}-\left(p_{1}+2 p_{2}\right)_{\mu_{1}} g_{\mu_{2} \mu_{3}}+\left(p_{2}+2 p_{1}\right)_{\mu_{2}} g_{\mu_{1} \mu_{3}}\right) \text {, } \\
& (i i i)_{123}=i\left(\frac{1}{(4 \pi)^{2} \varepsilon}\right) \frac{1}{12} N\left[e^{-i \omega\left(p_{1}, p_{2}\right)} \delta_{i_{1}}^{j_{2}} \delta_{i_{2}}^{j_{3}} \delta_{i_{3}}^{j_{1}}-e^{i \omega\left(p_{1}, p_{2}\right)} \delta_{i_{1}}^{j_{3}} \delta_{i_{2}}^{j_{1}} \delta_{i_{3}}^{j_{2}}\right] \times \\
& \left(\left(-p_{1}-2 p_{2}\right)_{\mu_{3}} g_{\mu_{1} \mu_{2}}+\left(2 p_{1}+p_{2}\right)_{\mu_{1}} g_{\mu_{2} \mu_{3}}+\left(p_{2}-p_{1}\right)_{\mu_{2}} g_{\mu_{1} \mu_{3}}\right) \text {. }
\end{aligned}
$$

Summing over permutations one obtains

$$
\begin{aligned}
(I)= & (i)_{123}+(i)_{231}+(i)_{312}= \\
& i\left(\frac{1}{(4 \pi)^{2} \varepsilon}\right)\left(\frac{9}{2}+\frac{3\left(\lambda^{\prime}-1\right)}{4}\right) N\left[e^{-i \omega\left(p_{1}, p_{2}\right)} \delta_{i_{1}}^{j_{2}} \delta_{i_{2}}^{j_{3}} \delta_{i_{3}}^{j_{1}}-e^{i \omega\left(p_{1}, p_{2}\right)} \delta_{i_{1}}^{j_{3}} \delta_{i_{2}}^{j_{1}} \delta_{i_{3}}^{j_{2}}\right] \times \\
& \left(\left(p_{1}-p_{2}\right)_{\mu_{3}} g_{\mu_{1} \mu_{2}}-\left(2 p_{1}+p_{2}\right)_{\mu_{2}} g_{\mu_{1} \mu_{3}}+\left(p_{1}+2 p_{2}\right)_{\mu_{1}} g_{\mu_{2} \mu_{3}}\right), \\
(I I)= & (i i)_{123}= \\
& i\left(\frac{1}{(4 \pi)^{2} \varepsilon}\right)\left(\frac{13}{4}+\frac{9\left(\lambda^{\prime}-1\right)}{4}\right) N\left[e^{-i \omega\left(p_{1}, p_{2}\right)} \delta_{i_{1}}^{j_{2}} \delta_{i_{2}}^{j_{3}} \delta_{i_{3}}^{j_{1}}-e^{i \omega\left(p_{1}, p_{2}\right)} \delta_{i_{1}}^{j_{3}} \delta_{i_{2}}^{j_{1}} \delta_{i_{3}}^{j_{2}}\right] \times
\end{aligned}
$$




$$
\begin{aligned}
& \left(\left(p_{2}-p_{1}\right)_{\mu_{3}} g_{\mu_{1} \mu_{2}}-\left(p_{1}+2 p_{2}\right)_{\mu_{1}} g_{\mu_{2} \mu_{3}}+\left(p_{2}+2 p_{1}\right)_{\mu_{2}} g_{\mu_{1} \mu_{3}}\right), \\
(I I I)= & (i i i)_{123}+(i i i)_{213}= \\
& i\left(\frac{1}{(4 \pi)^{2} \varepsilon}\right) \frac{1}{12} N\left[e^{-i \omega\left(p_{1}, p_{2}\right)} \delta_{i_{1}}^{j_{2}} \delta_{i_{2}}^{j_{3}} \delta_{i_{3}}^{j_{1}}-e^{i \omega\left(p_{1}, p_{2}\right)} \delta_{i_{1}}^{j_{3}} \delta_{i_{2}}^{j_{1}} \delta_{i_{3}}^{j_{2}}\right] \times \\
& \left(\left(p_{1}-p_{2}\right)_{\mu_{3}} g_{\mu_{1} \mu_{2}}+\left(p_{1}+2 p_{2}\right)_{\mu_{1}} g_{\mu_{2} \mu_{3}}+\left(-2 p_{1}-p_{2}\right)_{\mu_{2}} g_{\mu_{1} \mu_{3}}\right) .
\end{aligned}
$$

The sum of $(I),(I I)$ and $(I I I)$ yields the UV divergent contribution to the 1PI 3-point function of the gauge field. Note that (I), (II) and (III) are proportional to the 3-point vertex of the gauge field.

\section{Appendix C. Gauge field 4-point function}

The UV divergent contribution to the 4-point 1PI function of the gauge field is computed by summing over the UV divergent parts of the diagrams, and the appropriate permutations of these diagrams, in Figure 5.

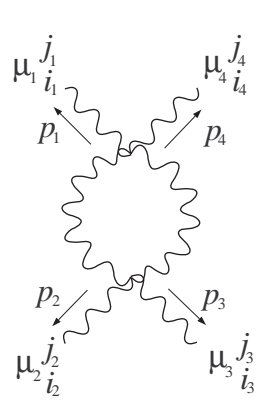

$(i)_{1234}$

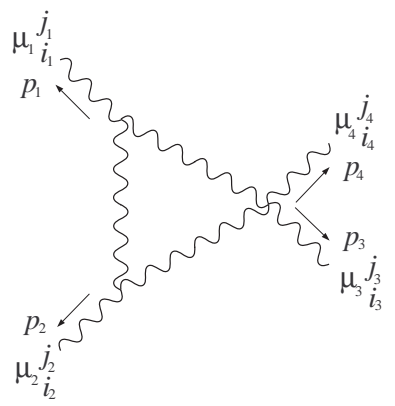

$(\text { ii })_{1234}$

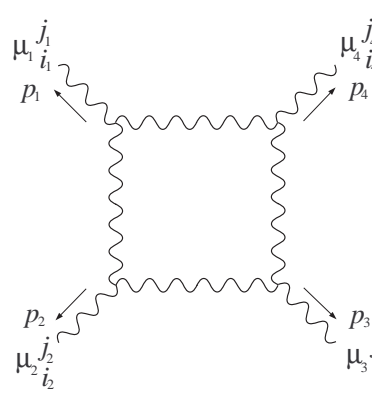

$(\text { iii })_{1234}$

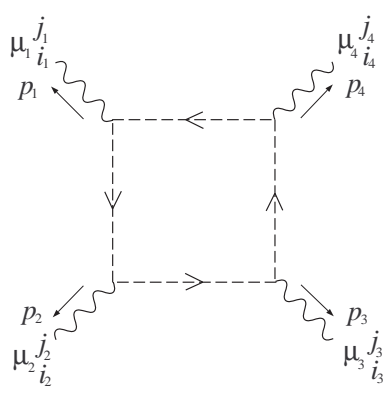

$(i v)_{1234}$

Figure 5: 1PI UV divergent 4-point Feynman diagrams for the gauge field.

The UV divergent part of the diagrams in Figure 5 read

$$
\begin{aligned}
(i)_{1234}= & i\left(\frac{1}{(4 \pi)^{2} \varepsilon}\right) N \times \\
& \left\{\left(e^{i\left[-\omega\left(p_{1}, p_{2}\right)-\omega\left(p_{1}, p_{3}\right)+\omega\left(p_{2}, p_{3}\right)\right]} \delta_{i_{1}}^{j_{3}} \delta_{i_{2}}^{j_{4}} \delta_{i_{3}}^{j_{2}} \delta_{i_{4}}^{j_{1}}+e^{i\left[\omega\left(p_{1}, p_{2}\right)+\omega\left(p_{1}, p_{3}\right)-\omega\left(p_{2}, p_{3}\right)\right]} \delta_{i_{1}}^{j_{4}} \delta_{i_{2}}^{j_{3}} \delta_{i_{3}}^{j_{1}} \delta_{i_{4}}^{j_{2}}\right)\right. \\
& {\left[\left(2+\left(\lambda^{\prime}-1\right)+\frac{13\left(\lambda^{\prime}-1\right)^{2}}{24}\right) g_{\mu_{1} \mu_{4}} g_{\mu_{2} \mu_{3}}+\right.} \\
& \left(5+\frac{5\left(\lambda^{\prime}-1\right)}{2}+\frac{\left(\lambda^{\prime}-1\right)^{2}}{24}\right) g_{\mu_{1} \mu_{3}} g_{\mu_{2} \mu_{4}}+
\end{aligned}
$$




$$
\begin{gathered}
\left.\left(-4-2\left(\lambda^{\prime}-1\right)+\frac{\left(\lambda^{\prime}-1\right)^{2}}{24}\right) g_{\mu_{1} \mu_{2}} g_{\mu_{3} \mu_{4}}\right]+ \\
\left(e^{i\left[-\omega\left(p_{1}, p_{2}\right)-\omega\left(p_{1}, p_{3}\right)-\omega\left(p_{2}, p_{3}\right)\right]} \delta_{i_{1}}^{j_{2}} \delta_{i_{2}}^{j_{3}} \delta_{i_{3}}^{j_{4}} \delta_{i_{4}}^{j_{1}}+e^{i\left[\omega\left(p_{1}, p_{2}\right)+\omega\left(p_{1}, p_{3}\right)+\omega\left(p_{2}, p_{3}\right)\right]} \delta_{i_{1}}^{j_{4}} \delta_{i_{2}}^{j_{1}} \delta_{i_{3}}^{j_{2}} \delta_{i_{4}}^{j_{3}}\right) \\
{\left[\left(2+\left(\lambda^{\prime}-1\right)+\frac{13\left(\lambda^{\prime}-1\right)^{2}}{24}\right) g_{\mu_{1} \mu_{4}} g_{\mu_{2} \mu_{3}}+\right.} \\
\quad\left(-4-2\left(\lambda^{\prime}-1\right)+\frac{\left(\lambda^{\prime}-1\right)^{2}}{24}\right) g_{\mu_{1} \mu_{3}} g_{\mu_{2} \mu_{4}}+ \\
\left.\left.\left(5+\frac{5\left(\lambda^{\prime}-1\right)}{2}+\frac{\left(\lambda^{\prime}-1\right)^{2}}{24}\right) g_{\mu_{1} \mu_{2}} g_{\mu_{3} \mu_{4}}\right]\right\},
\end{gathered}
$$

$(i i)_{1234}=i\left(\frac{1}{(4 \pi)^{2} \varepsilon}\right) N \times$

$$
\begin{aligned}
& \left\{\left(e^{i\left[\omega\left(p_{1}, p_{2}\right)-\omega\left(p_{1}, p_{3}\right)-\omega\left(p_{2}, p_{3}\right)\right]} \delta_{i_{1}}^{j_{3}} \delta_{i_{2}}^{j_{1}} \delta_{i_{3}}^{j_{4}} \delta_{i_{4}}^{j_{2}}+e^{i\left[-\omega\left(p_{1}, p_{2}\right)+\omega\left(p_{1}, p_{3}\right)+\omega\left(p_{2}, p_{3}\right)\right]} \delta_{i_{1}}^{j_{2}} \delta_{i_{2}}^{j_{4}} \delta_{i_{3}}^{j_{1}} \delta_{i_{4}}^{j_{3}}\right)\right. \\
& {\left[\left(2+\frac{17\left(\lambda^{\prime}-1\right)}{8}-\frac{\left(\lambda^{\prime}-1\right)^{2}}{24}\right) g_{\mu_{1} \mu_{4}} g_{\mu_{2} \mu_{3}}+\right.} \\
& \left(-\frac{5}{2}-\frac{19\left(\lambda^{\prime}-1\right)}{8}-\frac{\left(\lambda^{\prime}-1\right)^{2}}{24}\right) g_{\mu_{1} \mu_{3}} g_{\mu_{2} \mu_{4}}+ \\
& \left.\left(-\frac{13}{4}-\frac{13\left(\lambda^{\prime}-1\right)}{8}-\frac{13\left(\lambda^{\prime}-1\right)^{2}}{24}\right) g_{\mu_{1} \mu_{2}} g_{\mu_{3} \mu_{4}}\right]+ \\
& \left(e^{i\left[-\omega\left(p_{1}, p_{2}\right)-\omega\left(p_{1}, p_{3}\right)-\omega\left(p_{2}, p_{3}\right)\right]} \delta_{i_{1}}^{j_{2}} \delta_{i_{2}}^{j_{3}} \delta_{i_{3}}^{j_{4}} \delta_{i_{4}}^{j_{1}}+e^{i\left[\omega\left(p_{1}, p_{2}\right)+\omega\left(p_{1}, p_{3}\right)+\omega\left(p_{2}, p_{3}\right)\right]} \delta_{i_{1}}^{j_{4}} \delta_{i_{2}}^{j_{1}} \delta_{i_{3}}^{j_{2}} \delta_{i_{4}}^{j_{3}}\right) \\
& {\left[\left(-\frac{5}{2}-\frac{19\left(\lambda^{\prime}-1\right)}{8}-\frac{\left(\lambda^{\prime}-1\right)^{2}}{24}\right) g_{\mu_{1} \mu_{4}} g_{\mu_{2} \mu_{3}}+\right.} \\
& \left(2+\frac{17\left(\lambda^{\prime}-1\right)}{8}-\frac{\left(\lambda^{\prime}-1\right)^{2}}{24}\right) g_{\mu_{1} \mu_{3}} g_{\mu_{2} \mu_{4}}+ \\
& \left.\left.\left(-\frac{13}{4}-\frac{13\left(\lambda^{\prime}-1\right)}{8}-\frac{13\left(\lambda^{\prime}-1\right)^{2}}{24}\right) g_{\mu_{1} \mu_{2}} g_{\mu_{3} \mu_{4}}\right]\right\} \text {, }
\end{aligned}
$$

$(\text { iii })_{1234}=i\left(\frac{1}{(4 \pi)^{2} \varepsilon}\right) N \times$

$$
\begin{gathered}
\left\{\left(e^{i\left[-\omega\left(p_{1}, p_{2}\right)-\omega\left(p_{1}, p_{3}\right)-\omega\left(p_{2}, p_{3}\right)\right]} \delta_{i_{1}}^{j_{2}} \delta_{i_{2}}^{j_{3}} \delta_{i_{3}}^{j_{4}} \delta_{i_{4}}^{j_{1}}+e^{i\left[\omega\left(p_{1}, p_{2}\right)+\omega\left(p_{1}, p_{3}\right)+\omega\left(p_{2}, p_{3}\right)\right]} \delta_{i_{1}}^{j_{4}} \delta_{i_{2}}^{j_{1}} \delta_{i_{3}}^{j_{2}} \delta_{i_{4}}^{j_{3}}\right)\right. \\
{\left[\left(\frac{47}{12}+\frac{5\left(\lambda^{\prime}-1\right)}{2}+\frac{7\left(\lambda^{\prime}-1\right)^{2}}{12}\right) g_{\mu_{1} \mu_{4}} g_{\mu_{2} \mu_{3}}+\right.} \\
\quad\left(\frac{17}{12}-\frac{\left(\lambda^{\prime}-1\right)}{2}+\frac{\left(\lambda^{\prime}-1\right)^{2}}{12}\right) g_{\mu_{1} \mu_{3}} g_{\mu_{2} \mu_{4}}+
\end{gathered}
$$




$$
\begin{aligned}
& \left.\left.\left(\frac{47}{12}+\frac{5\left(\lambda^{\prime}-1\right)}{2}+\frac{7\left(\lambda^{\prime}-1\right)^{2}}{12}\right) g_{\mu_{1} \mu_{2}} g_{\mu_{3} \mu_{4}}\right]\right\}, \\
(i v)_{1234}=- & \frac{i}{24}\left(\frac{1}{(4 \pi)^{2} \varepsilon}\right) N \times \\
& \left(e^{i\left[-\omega\left(p_{1}, p_{2}\right)-\omega\left(p_{1}, p_{3}\right)-\omega\left(p_{2}, p_{3}\right)\right]} \delta_{i_{1}}^{j_{2}} \delta_{i_{2}}^{j_{3}} \delta_{i_{3}}^{j_{4}} \delta_{i_{4}}^{j_{1}}+e^{i\left[\omega\left(p_{1}, p_{2}\right)+\omega\left(p_{1}, p_{3}\right)+\omega\left(p_{2}, p_{3}\right)\right]} \delta_{i_{1}}^{j_{4}} \delta_{i_{2}}^{j_{1}} \delta_{i_{3}}^{j_{2}} \delta_{i_{4}}^{j_{3}}\right) \\
& \left(g_{\mu_{1} \mu_{4}} g_{\mu_{2} \mu_{3}}+g_{\mu_{1} \mu_{3}} g_{\mu_{2} \mu_{4}}+g_{\mu_{1} \mu_{2}} g_{\mu_{3} \mu_{4}}\right) .
\end{aligned}
$$

Summing over permutations, one obtains

$$
\begin{aligned}
& (I)=(i)_{1234}+(i)_{3214}+(i)_{2134}= \\
& =i\left(\frac{1}{(4 \pi)^{2} \varepsilon}\right) N \times \\
& \left\{\left(e^{i\left[-\omega\left(p_{1}, p_{2}\right)-\omega\left(p_{1}, p_{3}\right)+\omega\left(p_{2}, p_{3}\right)\right]} \delta_{i_{1}}^{j_{3}} \delta_{i_{2}}^{j_{4}} \delta_{i_{3}}^{j_{2}} \delta_{i_{4}}^{j_{1}}+e^{i\left[\omega\left(p_{1}, p_{2}\right)+\omega\left(p_{1}, p_{3}\right)-\omega\left(p_{2}, p_{3}\right)\right]} \delta_{i_{1}}^{j_{4}} \delta_{i_{2}}^{j_{3}} \delta_{i_{3}}^{j_{1}} \delta_{i_{4}}^{j_{2}}\right)\right. \\
& {\left[\left(7+\frac{7\left(\lambda^{\prime}-1\right)}{2}+\frac{7\left(\lambda^{\prime}-1\right)^{2}}{12}\right) g_{\mu_{1} \mu_{4}} g_{\mu_{2} \mu_{3}}+\right.} \\
& \left(7+\frac{7\left(\lambda^{\prime}-1\right)}{2}+\frac{7\left(\lambda^{\prime}-1\right)^{2}}{12}\right) g_{\mu_{1} \mu_{3}} g_{\mu_{2} \mu_{4}}+ \\
& \left.\left(-8-4\left(\lambda^{\prime}-1\right)+\frac{\left(\lambda^{\prime}-1\right)^{2}}{12}\right) g_{\mu_{1} \mu_{2}} g_{\mu_{3} \mu_{4}}\right]+ \\
& \left(e^{i\left[-\omega\left(p_{1}, p_{2}\right)-\omega\left(p_{1}, p_{3}\right)-\omega\left(p_{2}, p_{3}\right)\right]} \delta_{i_{1}}^{j_{2}} \delta_{i_{2}}^{j_{3}} \delta_{i_{3}}^{j_{4}} \delta_{i_{4}}^{j_{1}}+e^{i\left[\omega\left(p_{1}, p_{2}\right)+\omega\left(p_{1}, p_{3}\right)+\omega\left(p_{2}, p_{3}\right)\right]} \delta_{i_{1}}^{j_{4}} \delta_{i_{2}}^{j_{1}} \delta_{i_{3}}^{j_{2}} \delta_{i_{4}}^{j_{3}}\right) \\
& {\left[\left(7+\frac{7\left(\lambda^{\prime}-1\right)}{2}+\frac{7\left(\lambda^{\prime}-1\right)^{2}}{12}\right) g_{\mu_{1} \mu_{4}} g_{\mu_{2} \mu_{3}}+\right.} \\
& \left(-8-4\left(\lambda^{\prime}-1\right)+\frac{\left(\lambda^{\prime}-1\right)^{2}}{12}\right) g_{\mu_{1} \mu_{3}} g_{\mu_{2} \mu_{4}}+ \\
& \left.\left(7+\frac{7\left(\lambda^{\prime}-1\right)}{2}+\frac{7\left(\lambda^{\prime}-1\right)^{2}}{12}\right) g_{\mu_{1} \mu_{2}} g_{\mu_{3} \mu_{4}}\right]+ \\
& \left(e^{i\left[\omega\left(p_{1}, p_{2}\right)-\omega\left(p_{1}, p_{3}\right)-\omega\left(p_{2}, p_{3}\right)\right]} \delta_{i_{1}}^{j_{3}} \delta_{i_{2}}^{j_{1}} \delta_{i_{3}}^{j_{4}} \delta_{i_{4}}^{j_{2}}+e^{i\left[-\omega\left(p_{1}, p_{2}\right)+\omega\left(p_{1}, p_{3}\right)+\omega\left(p_{2}, p_{3}\right)\right]} \delta_{i_{1}}^{j_{2}} \delta_{i_{2}}^{j_{4}} \delta_{i_{3}}^{j_{1}} \delta_{i_{4}}^{j_{3}}\right) \\
& {\left[\left(-8-4\left(\lambda^{\prime}-1\right)+\frac{\left(\lambda^{\prime}-1\right)^{2}}{12}\right) g_{\mu_{1} \mu_{4}} g_{\mu_{2} \mu_{3}}+\right.} \\
& \left(7+\frac{7\left(\lambda^{\prime}-1\right)}{2}+\frac{7\left(\lambda^{\prime}-1\right)^{2}}{12}\right) g_{\mu_{1} \mu_{3}} g_{\mu_{2} \mu_{4}}+ \\
& \left.\left.\left(7+\frac{7\left(\lambda^{\prime}-1\right)}{2}+\frac{7\left(\lambda^{\prime}-1\right)^{2}}{12}\right) g_{\mu_{1} \mu_{2}} g_{\mu_{3} \mu_{4}}\right]\right\} \\
& (I I)=(i i)_{1234}+(i i)_{1324}+(i i)_{1423}+(i i)_{2314}+(i i)_{2413}+(i i)_{3412}= \\
& =i\left(\frac{1}{(4 \pi)^{2} \varepsilon}\right) N \times
\end{aligned}
$$




$$
\begin{aligned}
& \left\{\left(e^{i\left[\omega\left(p_{1}, p_{2}\right)-\omega\left(p_{1}, p_{3}\right)-\omega\left(p_{2}, p_{3}\right)\right]} \delta_{i_{1}}^{j_{3}} \delta_{i_{2}}^{j_{1}} \delta_{i_{3}}^{j_{4}} \delta_{i_{4}}^{j_{2}}+e^{i\left[-\omega\left(p_{1}, p_{2}\right)+\omega\left(p_{1}, p_{3}\right)+\omega\left(p_{2}, p_{3}\right)\right]} \delta_{i_{1}}^{j_{2}} \delta_{i_{2}}^{j_{4}} \delta_{i_{3}}^{j_{1}} \delta_{i_{4}}^{j_{3}}\right)\right. \\
& {\left[\left(8+\frac{17\left(\lambda^{\prime}-1\right)}{2}-\frac{\left(\lambda^{\prime}-1\right)^{2}}{6}\right) g_{\mu_{1} \mu_{4}} g_{\mu_{2} \mu_{3}}+\right.} \\
& \left(-\frac{23}{2}-8\left(\lambda^{\prime}-1\right)-\frac{7\left(\lambda^{\prime}-1\right)^{2}}{6}\right) g_{\mu_{1} \mu_{3}} g_{\mu_{2} \mu_{4}}+ \\
& \left.\left(-\frac{23}{2}-8\left(\lambda^{\prime}-1\right)-\frac{7\left(\lambda^{\prime}-1\right)^{2}}{6}\right) g_{\mu_{1} \mu_{2}} g_{\mu_{3} \mu_{4}}\right]+ \\
& \left(e^{i\left[-\omega\left(p_{1}, p_{2}\right)-\omega\left(p_{1}, p_{3}\right)-\omega\left(p_{2}, p_{3}\right)\right]} \delta_{i_{1}}^{j_{2}} \delta_{i_{2}}^{j_{3}} \delta_{i_{3}}^{j_{4}} \delta_{i_{4}}^{j_{1}}+e^{i\left[\omega\left(p_{1}, p_{2}\right)+\omega\left(p_{1}, p_{3}\right)+\omega\left(p_{2}, p_{3}\right)\right]} \delta_{i_{1}}^{j_{4}} \delta_{i_{2}}^{j_{1}} \delta_{i_{3}}^{j_{2}} \delta_{i_{4}}^{j_{3}}\right) \\
& {\left[\left(-\frac{23}{2}-8\left(\lambda^{\prime}-1\right)-\frac{7\left(\lambda^{\prime}-1\right)^{2}}{6}\right) g_{\mu_{1} \mu_{4}} g_{\mu_{2} \mu_{3}}+\right.} \\
& \left(8+\frac{17\left(\lambda^{\prime}-1\right)}{2}-\frac{\left(\lambda^{\prime}-1\right)^{2}}{6}\right) g_{\mu_{1} \mu_{3}} g_{\mu_{2} \mu_{4}}+ \\
& \left.\left(-\frac{23}{2}-8\left(\lambda^{\prime}-1\right)-\frac{7\left(\lambda^{\prime}-1\right)^{2}}{6}\right) g_{\mu_{1} \mu_{2}} g_{\mu_{3} \mu_{4}}\right]+ \\
& \left(e^{i\left[-\omega\left(p_{1}, p_{2}\right)-\omega\left(p_{1}, p_{3}\right)+\omega\left(p_{2}, p_{3}\right)\right]} \delta_{i_{1}}^{j_{3}} \delta_{i_{2}}^{j_{4}} \delta_{i_{3}}^{j_{2}} \delta_{i_{4}}^{j_{1}}+e^{i\left[\omega\left(p_{1}, p_{2}\right)+\omega\left(p_{1}, p_{3}\right)-\omega\left(p_{2}, p_{3}\right)\right]} \delta_{i_{1}}^{j_{4}} \delta_{i_{2}}^{j_{3}} \delta_{i_{3}}^{j_{1}} \delta_{i_{4}}^{j_{2}}\right) \\
& {\left[\left(-\frac{23}{2}-8\left(\lambda^{\prime}-1\right)-\frac{7\left(\lambda^{\prime}-1\right)^{2}}{6}\right) g_{\mu_{1} \mu_{4}} g_{\mu_{2} \mu_{3}}+\right.} \\
& \left(-\frac{23}{2}-8\left(\lambda^{\prime}-1\right)-\frac{7\left(\lambda^{\prime}-1\right)^{2}}{6}\right) g_{\mu_{1} \mu_{3}} g_{\mu_{2} \mu_{4}}+ \\
& \left.\left.\left(8+\frac{17\left(\lambda^{\prime}-1\right)}{2}-\frac{\left(\lambda^{\prime}-1\right)^{2}}{6}\right) g_{\mu_{1} \mu_{2}} g_{\mu_{3} \mu_{4}}\right]\right\} \text {, } \\
& (I I I)=(i i i)_{1234}+(\text { iii })_{1324}+(\text { iii })_{1243}= \\
& =i\left(\frac{1}{(4 \pi)^{2} \varepsilon}\right) N \times \\
& \left\{\left(e^{i\left[-\omega\left(p_{1}, p_{2}\right)-\omega\left(p_{1}, p_{3}\right)-\omega\left(p_{2}, p_{3}\right)\right]} \delta_{i_{1}}^{j_{2}} \delta_{i_{2}}^{j_{3}} \delta_{i_{3}}^{j_{4}} \delta_{i_{4}}^{j_{1}}+e^{i\left[\omega\left(p_{1}, p_{2}\right)+\omega\left(p_{1}, p_{3}\right)+\omega\left(p_{2}, p_{3}\right)\right]} \delta_{i_{1}}^{j_{4}} \delta_{i_{2}}^{j_{1}} \delta_{i_{3}}^{j_{2}} \delta_{i_{4}}^{j_{3}}\right)\right. \\
& {\left[\left(\frac{47}{12}+\frac{5\left(\lambda^{\prime}-1\right)}{2}+\frac{7\left(\lambda^{\prime}-1\right)^{2}}{12}\right) g_{\mu_{1} \mu_{4}} g_{\mu_{2} \mu_{3}}+\right.} \\
& \left(\frac{17}{12}-\frac{\left(\lambda^{\prime}-1\right)}{2}+\frac{\left(\lambda^{\prime}-1\right)^{2}}{12}\right) g_{\mu_{1} \mu_{3}} g_{\mu_{2} \mu_{4}}+ \\
& \left.\left(\frac{47}{12}+\frac{5\left(\lambda^{\prime}-1\right)}{2}+\frac{7\left(\lambda^{\prime}-1\right)^{2}}{12}\right) g_{\mu_{1} \mu_{2}} g_{\mu_{3} \mu_{4}}\right]+ \\
& \left(e^{i\left[-\omega\left(p_{1}, p_{2}\right)-\omega\left(p_{1}, p_{3}\right)+\omega\left(p_{2}, p_{3}\right)\right]} \delta_{i_{1}}^{j_{3}} \delta_{i_{2}}^{j_{4}} \delta_{i_{3}}^{j_{2}} \delta_{i_{4}}^{j_{1}}+e^{i\left[\omega\left(p_{1}, p_{2}\right)+\omega\left(p_{1}, p_{3}\right)-\omega\left(p_{2}, p_{3}\right)\right]} \delta_{i_{1}}^{j_{4}} \delta_{i_{2}}^{j_{3}} \delta_{i_{3}}^{j_{1}} \delta_{i_{4}}^{j_{2}}\right)
\end{aligned}
$$




$$
\begin{aligned}
& {\left[\left(\frac{47}{12}+\frac{5\left(\lambda^{\prime}-1\right)}{2}+\frac{7\left(\lambda^{\prime}-1\right)^{2}}{12}\right) g_{\mu_{1} \mu_{4}} g_{\mu_{2} \mu_{3}}+\right.} \\
& \left(\frac{47}{12}+\frac{5\left(\lambda^{\prime}-1\right)}{2}+\frac{7\left(\lambda^{\prime}-1\right)^{2}}{12}\right) g_{\mu_{1} \mu_{3}} g_{\mu_{2} \mu_{4}}+ \\
& \left.\left(\frac{17}{12}-\frac{\left(\lambda^{\prime}-1\right)}{2}+\frac{\left(\lambda^{\prime}-1\right)^{2}}{12}\right) g_{\mu_{1} \mu_{2}} g_{\mu_{3} \mu_{4}}\right]+ \\
& \left(e^{i\left[\omega\left(p_{1}, p_{2}\right)-\omega\left(p_{1}, p_{3}\right)-\omega\left(p_{2}, p_{3}\right)\right]} \delta_{i_{1}}^{j_{3}} \delta_{i_{2}}^{j_{1}} \delta_{i_{3}}^{j_{4}} \delta_{i_{4}}^{j_{2}}+e^{i\left[-\omega\left(p_{1}, p_{2}\right)+\omega\left(p_{1}, p_{3}\right)+\omega\left(p_{2}, p_{3}\right)\right]} \delta_{i_{1}}^{j_{2}} \delta_{i_{2}}^{j_{4}} \delta_{i_{3}}^{j_{1}} \delta_{i_{4}}^{j_{3}}\right) \\
& {\left[\left(\frac{17}{12}-\frac{\left(\lambda^{\prime}-1\right)}{2}+\frac{\left(\lambda^{\prime}-1\right)^{2}}{12}\right) g_{\mu_{1} \mu_{4}} g_{\mu_{2} \mu_{3}}+\right.} \\
& \left(\frac{47}{12}+\frac{5\left(\lambda^{\prime}-1\right)}{2}+\frac{7\left(\lambda^{\prime}-1\right)^{2}}{12}\right) g_{\mu_{1} \mu_{3}} g_{\mu_{2} \mu_{4}}+ \\
& \left.\left.\left(\frac{47}{12}+\frac{5\left(\lambda^{\prime}-1\right)}{2}+\frac{7\left(\lambda^{\prime}-1\right)^{2}}{12}\right) g_{\mu_{1} \mu_{2}} g_{\mu_{3} \mu_{4}}\right]\right\} \\
& (I V)=(i v)_{1234}+(i v)_{1324}+(i v)_{1243}+(i v)_{1432}+(i v)_{1342}+(i v)_{1423}= \\
& =-\frac{i}{12}\left(\frac{1}{(4 \pi)^{2} \varepsilon}\right) N \times \\
& \left(e^{i\left[-\omega\left(p_{1}, p_{2}\right)-\omega\left(p_{1}, p_{3}\right)-\omega\left(p_{2}, p_{3}\right)\right]} \delta_{i_{1}}^{j_{2}} \delta_{i_{2}}^{j_{3}} \delta_{i_{3}}^{j_{4}} \delta_{i_{4}}^{j_{1}}+e^{i\left[\omega\left(p_{1}, p_{2}\right)+\omega\left(p_{1}, p_{3}\right)+\omega\left(p_{2}, p_{3}\right)\right]} \delta_{i_{1}}^{j_{4}} \delta_{i_{2}}^{j_{1}} \delta_{i_{3}}^{j_{2}} \delta_{i_{4}}^{j_{3}}+\right. \\
& e^{i\left[-\omega\left(p_{1}, p_{2}\right)-\omega\left(p_{1}, p_{3}\right)+\omega\left(p_{2}, p_{3}\right)\right]} \delta_{i_{1}}^{j_{3}} \delta_{i_{2}}^{j_{4}} \delta_{i_{3}}^{j_{2}} \delta_{i_{4}}^{j_{1}}+e^{i\left[\omega\left(p_{1}, p_{2}\right)+\omega\left(p_{1}, p_{3}\right)-\omega\left(p_{2}, p_{3}\right)\right]} \delta_{i_{1}}^{j_{4}} \delta_{i_{2}}^{j_{3}} \delta_{i_{3}}^{j_{1}} \delta_{i_{4}}^{j_{2}}+ \\
& \left.e^{i\left[\omega\left(p_{1}, p_{2}\right)-\omega\left(p_{1}, p_{3}\right)-\omega\left(p_{2}, p_{3}\right)\right]} \delta_{i_{1}}^{j_{3}} \delta_{i_{2}}^{j_{1}} \delta_{i_{3}}^{j_{4}} \delta_{i_{4}}^{j_{2}}+e^{i\left[-\omega\left(p_{1}, p_{2}\right)+\omega\left(p_{1}, p_{3}\right)+\omega\left(p_{2}, p_{3}\right)\right]} \delta_{i_{1}}^{j_{2}} \delta_{i_{2}}^{j_{4}} \delta_{i_{3}}^{j_{1}} \delta_{i_{4}}^{j_{3}}\right) \\
& \left(g_{\mu_{1} \mu_{4}} g_{\mu_{2} \mu_{3}}+g_{\mu_{1} \mu_{3}} g_{\mu_{2} \mu_{4}}+g_{\mu_{1} \mu_{2}} g_{\mu_{3} \mu_{4}}\right) \text {. }
\end{aligned}
$$

The sum

$$
(I)+(I I)+(I I I)+(I V)
$$

is the UV divergent part of the 1PI 4-point gauge function in the MS scheme. Note that (I), (II), (III) and (IV) are not proportional to the tree-level 4-point vertex. 


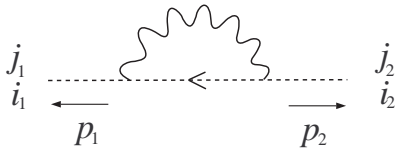

(i)

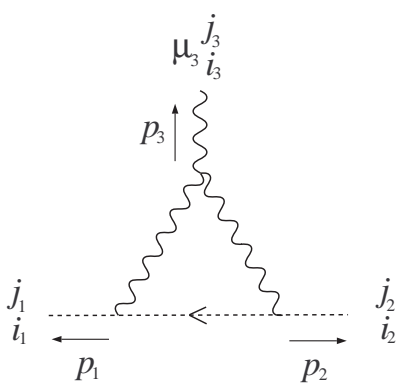

(ii)

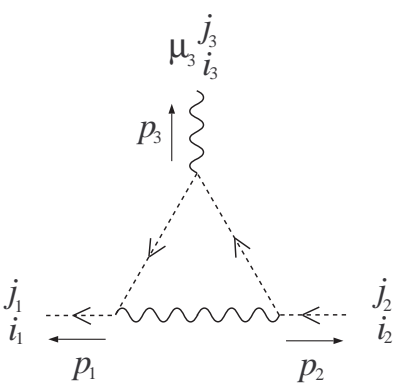

(iii)

Figure 6: 1PI UV divergent Feynman digrams for the ghost functions.

\section{Appendix D. Ghost functions}

The diagrams needed to compute the ghost self-energy and the ghost-boson vertex function are shown in Figure 6.

From them, we have obtained the following results

$$
\begin{aligned}
(i)= & -i\left(\frac{1}{(4 \pi)^{2} \varepsilon}\right) g^{2}\left(1-\frac{\lambda^{\prime}-1}{2}\right) N \delta_{i_{1}}^{j_{2}} \delta_{i_{2}}^{j_{1}} p_{1}^{2}, \\
(i i)= & 3 \frac{i}{4}\left(\frac{1}{(4 \pi)^{2} \varepsilon}\right) g^{2}\left(1+\left(\lambda^{\prime}-1\right)\right) \\
& N\left[e^{-i \omega\left(p_{1}, p_{2}\right)} \delta_{i_{1}}^{j_{2}} \delta_{i_{2}}^{j_{3}} \delta_{i_{3}}^{j_{1}}-e^{i \omega\left(p_{1}, p_{2}\right)}, \delta_{i_{1}}^{j_{3}} \delta_{i_{2}}^{j_{1}} \delta_{i_{3}}^{j_{2}}\right] p_{1 \mu_{3}}, \\
(i i i)= & \frac{i}{4}\left(\frac{1}{(4 \pi)^{2} \varepsilon}\right) g^{2}\left(1+\left(\lambda^{\prime}-1\right)\right) \\
& N\left[e^{-i \omega\left(p_{1}, p_{2}\right)} \delta_{i_{1}}^{j_{2}} \delta_{i_{2}}^{j_{3}} \delta_{i_{3}}^{j_{1}}-e^{i \omega\left(p_{1}, p_{2}\right)} \delta_{i_{1}}^{j_{3}} \delta_{i_{2}}^{j_{1}} \delta_{i_{3}}^{j_{2}}\right] p_{1 \mu_{3} .} .
\end{aligned}
$$

\section{Appendix E. Functions with external fields}

The diagrams which contribute at the one-loop level are shown in Figure 7. The results are:

$$
\begin{aligned}
(i)= & -i\left(\frac{1}{(4 \pi)^{2} \varepsilon}\right) g^{2} T_{R}\left(1-\frac{\lambda^{\prime}-1}{2}\right) N \delta_{i_{1}}^{j_{2}} \delta_{i_{2}}^{j_{1}} p_{1 \mu_{2}}, \\
(i i)= & -\frac{i}{4}\left(\frac{1}{(4 \pi)^{2} \varepsilon}\right) g^{2} T_{R}\left(1+\left(\lambda^{\prime}-1\right)\right) \times \\
& N\left[e^{-i \omega\left(p_{1}, p_{2}\right)} \delta_{i_{1}}^{j_{2}} \delta_{i_{2}}^{j_{3}} \delta_{i_{3}}^{j_{1}}-e^{i \omega\left(p_{1}, p_{2}\right)} \delta_{i_{1}}^{j_{3}} \delta_{i_{2}}^{j_{1}} \delta_{i_{3}}^{j_{2}}\right] g_{\mu_{2} \mu_{3}}, \\
(i i i)= & -\frac{3 i}{4}\left(\frac{1}{(4 \pi)^{2} \varepsilon}\right) g^{2} T_{R}\left(1+\left(\lambda^{\prime}-1\right)\right) \times
\end{aligned}
$$



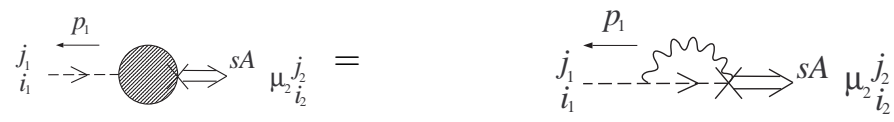

(i)

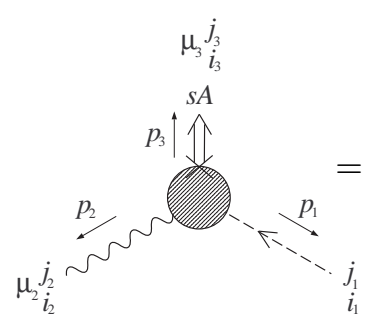

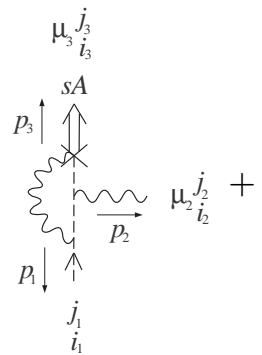

(ii)

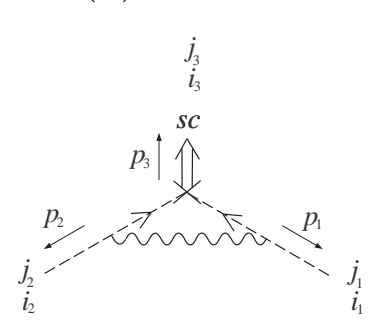

$(i v)$

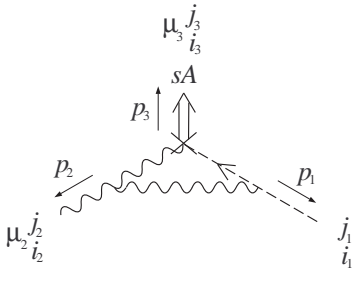

(iii)

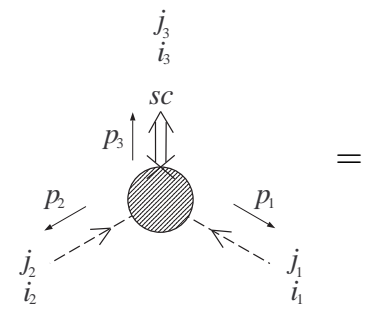

Figure 7: 1PI UV divergent digrams involving the external fields.

$$
\begin{gathered}
N\left[e^{-i \omega\left(p_{1}, p_{2}\right)} \delta_{i_{1}}^{j_{2}} \delta_{i_{2}}^{j_{3}} \delta_{i_{3}}^{j_{1}}-e^{i \omega\left(p_{1}, p_{2}\right)} \delta_{i_{1}}^{j_{3}} \delta_{i_{2}}^{j_{1}} \delta_{i_{3}}^{j_{2}}\right] g_{\mu_{2} \mu_{3}}, \\
(i v)=i\left(\frac{1}{(4 \pi)^{2} \varepsilon}\right) g^{2} T_{R}\left(1+\left(\lambda^{\prime}-1\right)\right) N\left[e^{-i \omega\left(p_{1}, p_{2}\right)} \delta_{i_{1}}^{j_{2}} \delta_{i_{2}}^{j_{3}} \delta_{i_{3}}^{j_{1}}-e^{i \omega\left(p_{1}, p_{2}\right)} \delta_{i_{1}}^{j_{3}} \delta_{i_{2}}^{j_{1}} \delta_{i_{3}}^{j_{2}}\right] .
\end{gathered}
$$

\section{Acknowledgments}

This work has been partially supported by CICyT under grant PB98-0842.

\section{References}

[1] A. González-Arroyo and M. Okawa, Phys. Lett. B120 (1983) 174; T. Eguchi and R. Nakayama, Phys. Lett. B122 (1983) 59; A. González-Arroyo and M. Okawa, Phys. Rev. D27 (1983) 2397; A. González-Arroyo and C. P. Korthals-Altes, Phys. Lett. B131 (1983) 396.

[2] Y. Makeenko, Reduced Models and Noncommutative Gauge Theories, hep-th/000928.

[3] S. Doplicher, K. Fredenhagen and J.E. Roberts, Phys. Letts. B331 (1994) 39; S. Doplicher, K. Fredenhagen and J.E. Roberts, Commun. Math. Phys. 172 (1995) 187. 
[4] A. Connes, M.R. Douglas and A. Schwarz, J. High Energy. Phys. 02 (1998) 003; hepth/9711162.

[5] M.R. Douglas and C. Hull, J. High Energy Phys. 9802 (1998) 008; H. Li, Comments on Supersymmetric Yang-Mills Theory on a Noncommutative Torus, hep-th/9802052; R. Casalbuoni, Phys. Lett. B431 (1998)69; A. Schwarz, Nucl. Phys. B534 (1998)720; T. Kawano and K. Okuyama, Phys. Lett. B433 (1998)29; Y.-K Cheung and M. Krog, Nucl. Phys. B528(1998)185 F. Ardalan, H. Arfaei and M.M. Sheikh-Jabbari, hepth/9803067; G. Landi, F. Lizzi and R.J. Szabo, Commun.Math.Phys. 206 (1999) 603; B. Moriaru and B. Zumino, hep-th/9807198; C. Hofman and E. Verlinde, Nucl. Phys. B547 (1999) 157.

[6] C.-S. Chu and P.-H. Ho, Nucl. Phys. B550 (1999) 151; F. Ardalan, H. Arfei and M.M. Sheikh-Jabbari, J. High Energy Phys. 02 (1999); V. Schomerus, J. High Energy Phys. 06 (1999) 030; L. Cornalba, D-brane Physics and Noncommutative Yang-Mills Theory, hep-th/9909091; N. Seiberg and E. Witten, J. High Energy Phys. 09 (1999) 032; O. Andreev and H. Dorn, Nucl. Phys. B583 (2000) 145; A. Bilal, C.-S. Chu and R. Russo, B582 (2000) 65; Y. Kien and S. Lee, Nucl. Phys. B586 (2000) 303; H. Liu and J. Michelson, Phys. Rev. D62 (2000) 066003.

[7] T. Filk, Phys. Lett. B376 (1996)53.

[8] A very incomplete list of references reads: N. Nekrasov and A.S. Schwarz, Comm. Math. Phys. 198 (1998) 689; J.C. Várilly and J.M. Gracía-Bondia, Int.J.Mod.Phys. A14 (1999) 1305; M. Chaichian, A. Demichev and P. Prešnajder, Nucl.Phys. B567 (2000) 360; P. Kosiński, J. Lukierski and P. Maślanka, Phys.Rev. D62 (2000) 025004; T. Krajewski and R. Wulkenhaar, Int.J.Mod.Phys. A15 (2000) 1011; C. P. Martín and D. Sánchez-Ruiz, Phys. Rev. Lett. 83 (1999) 476. M. Sheikh-Jabbari, J. High Energy Phys. 06 (1999) 015. S. Cho, R. Hinderting, J. Madore and H. Steinacker, Int. J. Mod. Phys. D9 (2000) 161; I. Y. Aref'eva, D.M. Belov and A.S Koshelev, Phys.Lett. B476 (2000) 431; C.-S Chu, Nucl.Phys. B580 (2000) 352; B. A. Campbell and K. Kaminsky, Nucl.Phys. B581 (2000) 240; F. Zamora, J. High Energy Phys. 0005 (2000) 002; W. Fischler, E. Gorbatov, A. Kashani-Poor, R. McNees, S. Paban and P. Pouliot, J. High Energy Phys. 0006 (2000) 032; G. Arcioni, J.L.F. Barbon, Joaquim Gomis and M.A. Vazquez-Mozo, J. High Energy Phys. 0006 (2000) 038; K. Furuta and T. Inami, Mod. Phys. Lett. A15 (2000) 997; G.-H. Chen and Y.-S Wu, One Loop Shift in Noncommutative Chern-Simons Coupling, hep-th/0006114; J. Gomis, T. Mehen and M. B. Wise, J. High Energy Phys. 0008 (2000) 029; Phys.Lett. B491 (2000) 345; L. Alvarez-Gaume and J.L.F. Barbon, Nonlinear Vacuum Phenomena in Noncommutative QED, hep-th/0006209 ; K. Landsteiner, E. Lopez and M.G.H. Tytgat, J. High Energy Phys. 0009 (2000) 027; J. W. Moffat, Phys.Lett. B491 (2000) 345; E. F. Moreno and F.A. Schaposnik, Wess-Zumino-Witten and Fermion Models in Noncommutative Space, hep-th/0008118; I.F. Riad and M. M. Sheikh-Jabbari, J. High Energy Phys. 0008 (2000) 045; S. Elitzur, B. Pioline and E. Rabinovici, J. High Energy Phys. 0010 (2000) 011; D.J. Gross, A. Hashimoto and N. Itzhaki, Observables of Noncommutative Gauge Theories, hep-th/0008075 W.-H. Huang, Two Loop Effective Potential in Noncommutative Scalar Field Theory, hep-th/0009067 ; N. Grandi and G.A. Silva, Chern-Simons action in Noncommutative Space, hep-th/0010113; 
H. Arfaei and M.H. Yavartanoo, Phenomenological Consequences of Noncommuative QED,hep-th/0010244; T. Mehen and M. B. Wise, Generalized *-Products, Wilson lines and the solution of the Seiberg-Witten Equations, hep-th/0010204; W.-H Huang, Finite Temperature Casimir Effect and the Radius Stabilization of the Noncommutative Torus, hep-th/0011037; M. Pernici, A. Santambrogio and D. Zanon, The one-loop effective action of noncommutative $\mathcal{N}=4$ super Yang-Mills is gauge invariant, hepth/0011140; D. Zanon, Noncommutative $\mathcal{N}=1,2$ super $U(N)$ Yang-Mills: $U V / I R$ mixing and effective action results at one loop, hep-th/0012009 .

[9] J. Ambjorn, Y.M. Makeenko, J. Nishimura and R.J. Szabo, Phys. Lett. B480 (2000) 399; J. High Energy Phys. 0005 (2000) 023.

[10] Irina Mocioiu, Maxim Pospelov and Radu Roiban, Phys. Lett. B489 (2000) 390; J.L. Hewett, F.J. Petriello and T.G. Rizzo, Signals for Non-Commutative Interactions at Linear Colliders, hep-ph/0010354; C.-S. Chu, B.R. Greene and G. Shiu, Remarks on Inflation and Noncommutative Geometry, hep-th/0011241; Prakash Mathews, Compton scattering in Noncommutative Space-Time at the NLC, hep-ph/0011332.

[11] S. Minwalla, M. Van Raamsdonk and N. Seiberg, Noncommutative Perturbative Dynamics, hep-th/9912027.

[12] M. Hayakawa, Phys. Lett. B478 (2000) 394; A. Matusis, L. Susskind and N. Toumbas, The IR/UV Connection in the Non-Commutative Gauge Theories, hep-th/0002075; J. Gomis, K. Landsteiner, E. Lopez, Phys.Rev. D62 (2000) 105006; J. Gomis, T. Mehen, M. B. Wise, J. High Energy Phys. 0008 (2000) 029; C.P. Martín and F. Ruiz Ruiz, Paramagnetic dominance, the sign of the beta function and UV/IR mixing in non-commutative $U(1)$, hep-th/0007131.

[13] I. Chepelev and R. Roiban, J. High Energy Phys. 0005 (2000) 037; Convergence Theorem for Non-commutative Feynman Graphs and Renormalization, hep-th/0008090.

[14] N.N. Bogoliubov and O.S. Parasiuk, Acta Math. 97 (1957) 227; K. Hepp, Comm. Math, Phys. 2 (1966) 301; W. Zimmerman Local Operator Products and Renormalization in Quantum Field Theory, 1970 Brandeis University Summer Institute in Thoeretical Physics, The M.I.T. Press, Edited by S. Deser, M. Grisaru and H. Pendleton.

[15] H.O. Girotti, M. Gomes, V.O. Rivelles and A.J. da Silva, Nucl.Phys. B587 (2000) 299; A.A. Bichl, J.M. Grimstrup, H. Grosse, L. Popp, M. Schweda and R. Wulkenhaar, The Superfield Formalism Applied to the Noncommutative Wess-Zumino Model, hepth/0007050.

[16] K. G. Wilson, Phys. Rev. B4 (1971) 3174; Phys. Rev. B4 (1971) 3184; J. Polchinski, Nuc. Phys B231 (1984) 269.

[17] S.S. Gubser and S. L. Sondhi, Phase structure of non-commutative scalar field theories, hep-th/0006119.

[18] I. Ya. Aref'eva, D. M. Belov and A. S.Koshelev, A Note on UV/IR for Noncommutative Complex Scalar Field, hep-th/0003176.

[19] J.H. Lowenstein, Phys. Rev. D4 (1971) 2281; Comm. Math. Phys. 24 (1971) 1; Y.M.P. Lam, Phys. Rev. D6 (1972) 2145; Phys. Rev. D7 (1973) 2943; T.E. Clark and J.H. Lowenstein, Nucl. Phys. B113 (1976) 109. 
[20] O. Piguet and S.P. Sorella, Algebraic Renormalization, Lectures notes in Physics, Monographs, Springer-Verlag 1995; J. Gomis, J. Paris and S. Samuel, Phys. Rep 259 (1995) 1; G. Barnich, F. Brandt and M. Henneaux, Phys. Rep. 338 (2000) 439.

[21] J. Gomis and T. Mehen, Nucl. Phys. B591 (2000) 265; O. Aharony, J. Gomis and T. Mehen, J. High Energy Phys. 0009 (2000) 023.

[22] J.M. Gracia-Bondía, J.C. Várilly and H. Figueroa, Elements of Noncommutative Geometry, Birkhauser, 2000; G. Landi, An Introduction to Noncommutative Spaces and their Geometries, Springer, Lecture Notes in Physics 51, Springer Verlag, 1997; J. Madore, An Introduction to Noncommutative Geometry and its applications, LMS, Cambridge University Press, 1995; A. Connes, Noncommutative Geometry, Academic Press, 1994.

[23] G. 't Hooft, Nucl. Phys. B72 (1974) 461.

[24] P. Breitenlohner and Maison, Comm. Math. Phys. 52 (1977) 11, ibid. 52 (1977) 39, 52 (1977) 55.

[25] A. Armoni, Comments on Perturbative Dynamics of Non-Commutative Yang-Mills Theory, hep-th/0005208; L. Bonora and M. Salizzoni, Renormalization of noncommutative $U(N)$ gauge theories, hep-th/0011088. 